\title{
INTENSITY MAPPING OF Ly $\alpha$ EMISSION DURING THE EPOCH OF REIONIZATION
}

\author{
Marta B. Silva ${ }^{1,2}$, Mario G. Santos ${ }^{1}$, Yan Gong ${ }^{2}$, Asantha Cooray ${ }^{2}$, and James Bock ${ }^{3,4}$ \\ ${ }^{1}$ CENTRA, Instituto Superior Técnico, Technical University of Lisbon, Lisboa 1049-001, Portugal \\ ${ }^{2}$ Department of Physics \& Astronomy, University of California, Irvine, CA 92697, USA \\ ${ }^{3}$ Department of Physics, Mathematics and Astronomy, California Institute of Technology, Pasadena, CA 91125, USA \\ ${ }^{4}$ Jet Propulsion Laboratory (JPL), National Aeronautics and Space Administration (NASA), Pasadena, CA 91109, USA \\ Received 2012 May 10; accepted 2012 November 30; published 2013 January 17
}

\begin{abstract}
We calculate the absolute intensity and anisotropies of the Ly $\alpha$ radiation field present during the epoch of reionization. We consider emission from both galaxies and the intergalactic medium (IGM) and take into account the main contributions to the production of Ly $\alpha$ photons: recombinations, collisions, continuum emission from galaxies, and scattering of Lyn photons in the IGM. We find that the emission from individual galaxies dominates over the IGM with a total Ly $\alpha$ intensity (times frequency) of about (1.43-3.57) $\times 10^{-8} \mathrm{erg} \mathrm{s}^{-1} \mathrm{~cm}^{-2} \mathrm{sr}^{-1}$ at a redshift of 7. This intensity level is low, so it is unlikely that the Ly $\alpha$ background during reionization can be established by an experiment aiming at an absolute background light measurement. Instead, we consider Ly $\alpha$ intensity mapping with the aim of measuring the anisotropy power spectrum that has rms fluctuations at the level of $1 \times 10^{-16}$ [erg s$\left.~^{-1} \mathrm{~cm}^{-2} \mathrm{sr}^{-1}\right]^{2}$ at a few Mpc scales. These anisotropies could be measured with a spectrometer at near-IR wavelengths from 0.9 to $1.4 \mu \mathrm{m}$ with fields in the order of 0.5 to $1 \mathrm{deg}^{2}$. We recommend that existing ground-based programs using narrowband filters also pursue intensity fluctuations to study statistics on the spatial distribution of faint Ly $\alpha$ emitters. We also discuss the cross-correlation signal with $21 \mathrm{~cm}$ experiments that probe $\mathrm{H} \mathrm{I}$ in the IGM during reionization. A dedicated sub-orbital or space-based Ly $\alpha$ intensity mapping experiment could provide a viable complimentary approach to probe reionization, when compared to $21 \mathrm{~cm}$ experiments, and is likely within experimental reach.
\end{abstract}

Key words: cosmology: theory - diffuse radiation - large-scale structure of universe

Online-only material: color figures

\section{INTRODUCTION}

The epoch of reionization (EoR) is a crucial stage in the history of galaxy formation, signaling the birth of the first luminous objects, during which the universe went from completely neutral to almost completely ionized (Barkana \& Loeb 2001). This phase has been largely unexplored so far, although current observations suggest that it was reasonably extended (Komatsu et al. 2011; Fan et al. 2006) and a wide variety of observational avenues are being explored to probe it. In particular, the $21 \mathrm{~cm}$ line of neutral hydrogen is now understood to be a promising tool to study reionization and to understand the formation and evolution of galaxies during that epoch (see, e.g., Furlanetto et al. 2006). It is also now becoming clear that we need complimentary data in order to obtain extra insight into the sources of reionization. Such complimentary data could also aid in the interpretation of the $\mathrm{H}$ I signal by allowing ways to pursue cross-correlations and providing ways to reduce systematics and foregrounds encountered in $21 \mathrm{~cm}$ observations.

Recently, intensity mapping of other atomic and molecular lines at high redshifts, in particular CO and C II (Gong et al. 2011, 2012; Lidz et al. 2011; Visbal \& Loeb 2010), has been proposed as a probe of reionization. In this work, we study the viability of also using intensity mapping of the Ly $\alpha$ line as an additional probe. For this study, we include several Ly $\alpha$ emission mechanisms involving both individual sources of emission such as galaxies and the emission and scattering associated with the intergalactic medium (IGM).

We consider both the integrated intensity and anisotropies of the Ly $\alpha$ line and suggest the latter as a new probe of reionization. In particular, we suggest that it will be possible to measure the amplitude of the Ly $\alpha$ intensity fluctuations with a narrowband spectrometer either from the ground with a suppression of atmospheric lines or from the orbital/sub-orbital platform.

The Ly $\alpha$ line, corresponding to transitions between the second and first energy level of the hydrogen atom, has a rest wavelength of approximately $\lambda_{\text {Ly } \alpha}=1216 \AA$. The signal present during reionization is observable in near-IR wavelengths today. Existing imaging observations made with narrowband filters on $10 \mathrm{~m}$ class telescopes focus on individual galaxy detections and are limited to a handful of narrow atmospheric windows at nearIR wavelengths. Given the strength of the line, it has now been seen in galaxies at $z \approx 6.98$ (Iye et al. 2006), $z \approx 8.2$ (Salvaterra et al. 2009), and $z \approx 8.6$ (Lehnert et al. 2010), reaching well into the EoR.

Deep narrowband surveys of high-redshift Ly $\alpha$ emitters have led to detections of a sufficient number of galaxies at redshifts 5.7, 6.6, 7.0, and 7.7 to allow constraints on the bright end of the Ly $\alpha$ luminosity function (LF) and its redshift evolution (e.g., Ouchi et al. 2008; Ota et al. 2010; Taniguchi et al. 2005; Iye et al. 2006; Shibuya et al. 2012). Observations of the Ly $\alpha$ LF indicate a decrease in the Ly $\alpha$ intensity from redshift 5.7 to 7.0. This would require a strong evolution of the $\mathrm{Ly} \alpha$ emitter population, which is not predicted by most recent galaxy evolution models (Ota et al. 2010; Shibuya et al. 2012), or could be explained as the result of an increase in the fraction of IGM neutral hydrogen that would absorb or scatter Ly $\alpha$ photons from the observed galaxies (Haiman et al. 2000; Ota et al. 2008).

The scattering of $\operatorname{Ly} \alpha$ photons by neutral hydrogen in the interstellar medium (ISM) and the IGM is expected to disperse the photons in both frequency and direction (Santos 2004). Such scattering could considerably decrease the Ly $\alpha$ intensity 
per frequency bin from an individual galaxy, making the detection of most of the high-redshift galaxies impossible with current instruments. Exact calculations related to scattering are a difficult problem to solve analytically, and in simulations the scattering problem requires ray tracing of photons through the neutral medium in a simulation box (Zheng et al. 2010). While scattering makes individual galaxies dimmer, intensity mapping of the Ly $\alpha$ line at high redshifts can be an improvement over the usual experiments that make detections of $\mathrm{Ly} \alpha$ emission from point sources and are only sensible to the strongest Ly $\alpha$ emitters. These are likely to be some of the brightest starforming galaxies; however, any dust that is present in such galaxies, especially during the late stages of reionization, is likely to suppress the Ly $\alpha$ line. An experiment targeting the integrated emission will be able to measure all the sources of Ly $\alpha$ photons in a large region and will be sensitive to the extended, low surface brightness $\operatorname{Ly} \alpha$ emission that is now known to generally form around star-forming regions (e.g., Steidel et al. 2011; Zheng et al. 2011). The anisotropy power spectrum of Ly $\alpha$ intensity then would be a probe of the Ly $\alpha$ halos around star-forming galaxies present during reionization. The crosscorrelation with the $21 \mathrm{~cm}$ data could provide a direct test on the presence of neutral hydrogen in the extended Ly $\alpha$ halo.

The paper is organized as follows: In the next section we estimate the contribution to the $\operatorname{Ly} \alpha$ emission from galaxies. In Section 3 we analyze the contributions to the $\operatorname{Ly} \alpha$ emission from the IGM. In Section 4 we calculate the intensity of the Ly $\alpha$ signal, as well as its power spectrum, using a modified version of the code SimFast21 (Santos et al. 2010, 2011). In Section 5 we discuss the correlation of Ly $\alpha$ intensity maps with the $21 \mathrm{~cm}$ signal. Finally, in Section 6 we comment on the experimental feasibility of measuring the $\operatorname{Ly} \alpha$ intensity power spectrum.

\section{Ly $\alpha$ EMISSION FROM GALAXIES}

The observed Ly $\alpha$ flux is mainly the result of line emission from hydrogen recombinations and collisional excitations in the interstellar clouds or in the IGM powered, respectively, by UV emission or UV and X-ray emission from galaxies. High-energy photons emitted by stars ionize hydrogen that then recombines to emit a rich spectrum of lines including an Ly $\alpha$ photon (Gould \& Weinberg 1996; Fernandez \& Komatsu 2006). Moreover, the electron ejected during this ionization heats the ISM or the IGM, increasing the probability of Ly $\alpha$ photon emission caused by collisional excitation (Gould \& Weinberg 1996; Cantalupo et al. 2008). There is also a small contribution to the Ly $\alpha$ flux originated in the continuum emission from stars between the Ly $\alpha$ line and the Lyman limit (Chuzhoy \& Zheng 2007; Barkana \& Loeb 2005) plus Ly $\alpha$ from continuum free-free or free-bound emission, as well as two-photon emission during recombinations. This continuum will also make contributions to a given observation from lower redshifts besides the "Ly $\alpha$ " redshift (Cooray et al. 2012), which will confuse the Ly $\alpha$ signal. However, due to the smoothness of that continuum across frequency, we expect it should be possible to remove this contribution, for instance, by fitting a smooth polynomial in frequency for each pixel.

Another source of $\operatorname{Ly} \alpha$ emission in the universe is cooling of gas that has suffered infall into a dark matter halo potential well. Several studies show that much of this cooling is made in the form of Ly $\alpha$ emission (Haiman et al. 2000; Fardal et al. 2001; Dijkstra et al. 2006a, 2006b; Dayal et al. 2010; Latif et al. 2011). Cold gas is used by galaxies as fuel to form stars, so there is a relation between the star formation rate (SFR) of a galaxy and the Ly $\alpha$ flux emitted as gas cools in that galaxy.

Since emission of Ly $\alpha$ radiation is closely connected with the star formation, the contribution from the several mechanisms by which Ly $\alpha$ radiation is emitted in galaxies and in the IGM can be related to the SFR of individual galaxies or galaxy samples. In order to calculate the emission of $\mathrm{Ly} \alpha$ radiation from the IGM during the EoR, we also need to know the ionized fraction of hydrogen and the temperature of the gas in the IGM. Unfortunately, both these quantities are poorly constrained at $z \geqslant 6$ (Larson et al. 2011; Ouchi et al. 2010; Zahn et al. 2012). Since hydrogen ionization should be a consequence of stellar ionization/X-ray emission, we can in principle estimate it by following the SFR history and making sure that the resulting evolution of hydrogen ionized fraction is consistent with current constraints on the cosmic microwave background optical depth.

In order to obtain the SFR of galaxies at the high redshifts during the EoR, we make use of parameterizations that reproduce a correct reionization history. Our parameterizations are nonlinear in a similar way to the relations found in the Guo et al. (2011) and the De Lucia \& Blaizot (2007) galaxy catalogs derived, respectively, from the high-resolution Millennium II (Boylan-Kolchin et al. 2009) and Millennium I (Springel et al. 2005) simulations. Such relations, when available from observations, make an improvement on the models instead of relying purely on theoretical calculations and semi-numerical simulations to predict all of the observations (Mesinger \& Furlanetto 2007; Santos et al. 2010).

There are additional sources of radiation contributing to the Ly $\alpha$ emission, such as strong non-local sources of ionizing photons as expected from quasars, which would emit a large amount of energy in X-ray photons that would be able to ionize several neutral atoms, giving origin to a locally strong Ly $\alpha$ emission from recombinations. However, since the number of quasars is very small compared to the number of normal galaxies at the redshifts we are considering, we will neglect their contribution in the following calculations. We encourage future works on $\operatorname{Ly} \alpha$ intensity to see if the shape of the power spectrum and other statistics can be used to choose between reionization histories that involve both galaxies and quasars.

In the following sub-sections we discuss in more detail the four processes for Ly $\alpha$ emission from galaxies: recombinations, excitations/relaxations, gas cooling, and photon emission from continuum processes.

\subsection{Ly $\alpha$ Emission from Hydrogen Recombinations}

Assuming ionizing equilibrium, the number of recombinations in galaxies is expected to match the number of ionizing photons that are absorbed in the galaxy and does not escape into the IGM. Depending on the temperature and density of the gas, a fraction of the radiation due to these recombinations is emitted in the Ly $\alpha$ line.

In the interstellar gas, most of the neutral hydrogen is in dense clouds with column densities greater than $3 \times 10^{18} \mathrm{~cm}^{-2}$. These clouds are optically thick to Ly $\alpha$ radiation, and Lyman photons are scattered in the galaxy several times before escaping into the IGM. Such multiple scatterings increase the probability of absorption. Assuming that these clouds are spherical and that the gas temperature is of the order of $10^{4} \mathrm{~K}$, Gould \& Weinberg (1996) used atomic physics to study the probability of the Ly $\alpha$ emission per hydrogen recombination. They estimated that a fraction $f_{\text {rec }} \approx 66 \%$ of the hydrogen recombinations would result in the emission of an Ly $\alpha$ photon and that most of the other 
recombinations would result in two-photon emission. These fractions should change with the temperature and the shape of the cloud, but such variations are expected to be small. Other calculations yield fractions between $62 \%$ and $68 \%$ according to the conditions in the cloud. In this paper we have chosen to use a value of $f_{\text {rec }}=66 \%$ since the overall uncertainty on this number is lower than the uncertainty on the number of hydrogen recombinations.

The absorption of Ly $\alpha$ photons by dust is difficult to estimate and changes from galaxy to galaxy. Gould \& Weinberg (1996) estimated that for a cloud with a column density $N \sim 10^{19} \mathrm{~cm}^{-2}$ the dust in the galaxy absorbs a fraction $f_{\text {dust }} \approx 4 \%$ of the emitted $\operatorname{Ly} \alpha$ photons before they reach the galaxy virial radius; however, recent observations of high-redshift galaxies indicate a much higher $f_{\text {dust }}$. In this study, we will use a redshift parameterization for the fraction of $\operatorname{Ly} \alpha$ photons that are not absorbed by dust $f_{\mathrm{Ly} \alpha}=1-f_{\text {dust }}$ that is double the value predicted by the study made by Hayes et al. (2011):

$$
f_{\text {Ly } \alpha}(z)=C_{\text {dust }} \times 10^{-3}(1+z)^{\xi},
$$

where $C_{\text {dust }}=3.34$ and $\xi=2.57$. The Hayes et al. (2011) parameterization was made so that $f_{\mathrm{Ly} \alpha}$ gives the difference between observed Ly $\alpha$ luminosities and Ly $\alpha$ luminosities scaled from SFRs assuming that the Ly $\alpha$ photons emitted in galaxies are only originated in recombinations. The high-redshift observations used to estimate $f_{\text {Ly } \alpha}$ are only of massive stars, while the bulk of Ly $\alpha$ emission is originated in the low-mass stars that cannot be detected by current surveys. According to several studies (Forero-Romero et al. 2011), $f_{\mathrm{Ly} \alpha}$ decreases with halo mass, so it is possible that it is being underestimated in Hayes et al. (2011), which is why we decided to use a higher $f_{\text {Ly } \alpha}$. Our results can, however, be easily scaled to other $f_{\text {dust }}$ evolutions.

The number of Ly $\alpha$ photons emitted in a galaxy per second, $\dot{N}_{\text {Ly } \alpha}$, that reach its virial radius is therefore given by

$$
\dot{N}_{\text {Ly } \alpha}=A_{\text {He }} f_{\text {rec }} \times f_{\text {Ly } \alpha} \times\left(1-f_{\text {esc }}\right) \times \dot{N}_{\text {ion }},
$$

where $A_{\mathrm{He}}=\left(4-4 Y_{p}\right) /\left(4-3 Y_{p}\right)$ accounts for the fraction of photons that go into the ionization of helium $\left(Y_{p}\right.$ is the mass fraction of helium), $\dot{N}_{\text {ion }}$ is the rate of ionizing photons emitted by the stars in the galaxy, and $f_{\text {esc }}$ is the fraction of ionizing photons that escape the galaxy into the IGM.

The ionizing photon escape fraction depends on conditions inside each galaxy and is difficult to estimate, especially at high redshifts. The precise determination of its value is one of the major goals of future observations of high-redshift galaxies at $z \gtrsim 7$. This parameter can be measured from deep imaging observations or can be estimated from the equivalent widths of the hydrogen and helium Balmer lines. The ionizing photon escape fraction dependence with the galaxy mass and the SFR, as a function of redshift, has been estimated using simulations that make several assumptions about the intensity of this radiation and its absorption in the ISM. However, for the halo virial mass range, $10^{8}-10^{13} M_{\odot}$, and during the broad redshift range related to the EoR, there are no simulations that cover the full parameter space. Moreover, the limited simulations that exist do not always agree with each other (Gnedin et al. 2008; Wise \& Cen 2009; Fernández-Soto et al. 2003; Siana et al. 2007; Haardt \& Madau 2012). Razoumov \& Sommer-Larsen (2010) computed the escape fraction of $U V$ radiation for the redshift interval $z=4$ to $z=10$ and for halos of masses from $10^{7.8}$ to $10^{11.5} M_{\odot}$ using a high-resolution set of galaxies. Their simulations cover most of the parameter space needed for reionization-related calculations,
Table 1

Fits to the Escape Fraction of UV Radiation from Galaxies as a Function of Redshift (based on Razoumov \& Sommer-Larsen 2010)

\begin{tabular}{lccc}
\hline \hline$z$ & $\alpha$ & $\beta$ & $f_{\mathrm{esc}}\left(M=10^{10} M_{\odot}\right)$ \\
\hline 10.4 & $2.78 \times 10^{-2}$ & 0.105 & 0.732 \\
8.2 & $1.30 \times 10^{-2}$ & 0.179 & 0.449 \\
6.7 & $5.18 \times 10^{-3}$ & 0.244 & 0.240 \\
5.7 & $3.42 \times 10^{-3}$ & 0.262 & 0.240 \\
\hline
\end{tabular}

and their escape fraction parameterization is compatible with most of the current observational results. Thus, we use it for our calculations here.

According to Razoumov \& Sommer-Larsen (2010) simulations, the escape fraction of ionizing radiation can be parameterized as

$$
f_{\text {esc }}(M, z)=\exp \left[-\alpha(z) M^{\beta(z)}\right],
$$

where $M$ is the halo mass and $\alpha$ and $\beta$ are functions of redshift (Table 1).

The number of ionizing photons emitted by the stars in a galaxy depends on its SFR, metallicity, and the stellar initial mass function (IMF). Making reasonable assumptions for these quantities, we will now estimate $\dot{N}_{\text {ion }}$. Since this UV emission is dominated by massive, short-lived stars, we can assume that the intensity of ionizing photons emitted by a galaxy is proportional to its SFR. In terms of the SFR in one galaxy,

$$
\dot{N}_{\text {ion }}=Q_{\text {ion }} \times \mathrm{SFR},
$$

where $Q_{\text {ion }}$ is the average number of ionizing photons emitted per solar mass of star formation. This can be calculated through

$$
Q_{\text {ion }}=\frac{\int_{M_{\min }}^{M_{\max }} \Psi(M) Q_{\star}(M) t_{\star}(M) d M}{\int_{M_{\min }}^{M_{\max }} \Psi(M) M d M},
$$

where $\Psi(M)=K M^{-\alpha}$ is the stellar IMF, $K$ is a constant normalization factor, and $\alpha$ is the slope of the IMF. In our calculation, we used a Salpeter IMF, with $\alpha=2.35 . t_{\star}(M)$ is the star lifetime and $Q_{\star}(M)$ its number of ionizing photons emitted per unit time. The values of $Q_{\star}$ and $t_{\star}$ were calculated with the ionizing fluxes obtained by Schaerer (2002) using realistic models of stellar populations and non-LTE atmospheric models, appropriated for Pop II stars with a $Z_{\star}=0.02 Z_{\odot}$ metallicity.

Assuming that ionizing photons are only emitted by massive OB stars sets a low mass effective limit for the mass of stars contributing to the UV radiation field of a galaxy. This limit is a necessary condition for the star to be able to produce a significant number of ionizing photons. For the stellar population used for this work we take $M_{\min } \approx 7 M_{\odot}$ (Schaerer 2002; Shull et al. 2012). The integration upper limit is taken to be $M_{\max }=150 M_{\odot}$. In this paper, we calculated $Q_{\text {ion }}$ using the parameterization values published in Schaerer (2002). The number of ionizing photons per second emitted by a star as a function of its mass is given by

$$
\begin{aligned}
\log _{10}\left[Q_{\star} / \mathrm{s}^{-1}\right]= & 27.80+30.68 x-14.80 x^{2} \\
& +2.5 x^{3} \text { for } 7 M_{\odot}<M_{\star}<150 M_{\odot},
\end{aligned}
$$

where $x=\log _{10}\left(M_{\star} / M_{\odot}\right)$ and the star's lifetime in years is given by

$$
\log _{10}\left[t_{\star} / \mathrm{yr}\right]=9.59-2.79 x+0.63 x^{2} \text {. }
$$


The use of these parameters results in $Q_{\text {ion }} \approx 5.38 \times 10^{60} M_{\odot}^{-1}$. Shull et al. (2012) suggest the use of a different model for stellar atmosphere and evolution (R. S. Sutherland \& J. M. Shull, unpublished) that yields $Q_{\text {ion }} \approx 3.97 \times 10^{60} M_{\odot}^{-1}$. This may imply that the stellar emissivity we calculated is an overestimation and that consequently our Ly $\alpha$ flux powered by stellar emission may be overestimated by about $35 \%$. This is comparable to other large uncertainties, such as the ones in the parameters $f_{\text {esc }}$ and $f_{\text {dust }}$. The Ly $\alpha$ luminosity is calculated assuming that the Ly $\alpha$ photons are emitted at the Ly $\alpha$ rest frequency, $v_{0}=2.47 \times 10^{15} \mathrm{~Hz}$ with an energy of $E_{\mathrm{Ly} \alpha}=1.637 \times 10^{-11} \mathrm{erg}$. To proceed, we will assume that the SFR for a given galaxy is only a function of redshift and the mass of the dark halo associated with that galaxy. The Ly $\alpha$ luminosity due to recombinations in the ISM, $L_{\mathrm{rec}}^{\mathrm{GAL}}$, can then be parameterized as a function of halo mass and redshift as

$$
\begin{aligned}
L_{\mathrm{rec}}^{\mathrm{GAL}}(M, z)= & E_{\mathrm{Ly} \alpha} \dot{N}_{\mathrm{Ly} \alpha} \\
\approx & 1.55 \times 10^{42}\left[1-f_{\mathrm{esc}}(M, z)\right] f_{\mathrm{Ly} \alpha}(z) \\
& \times \frac{\operatorname{SFR}(M, z)}{M_{\odot} \mathrm{yr}^{-1}} \operatorname{erg~s}^{-1}
\end{aligned}
$$

\subsection{Ly $\alpha$ Emission from Excitations/Relaxations}

The kinetic energy of the electron ejected during the hydrogen ionization heats the gas, and assuming thermal equilibrium, this heat is emitted as radiation. Using atomic physics, Gould \& Weinberg (1996) estimated that for a cloud with a hydrogen column density of $\approx 10^{19} \mathrm{~cm}^{-2}$, the energy emitted in the form of Ly $\alpha$ photons is about $60 \%$ for ionizing photons with energy $E_{v_{\lim }}<E_{v}<4 E_{v_{\lim }}$ and $\approx 50 \%$ for photons with energy $E_{v}>4 E_{v_{\lim }}$, where $E_{v_{\lim }}=13.6 \mathrm{eV}$ is the Rydberg energy. The remaining energy is emitted in other lines.

Using the spectral energy distribution (SED) of galaxies with a metallicity $Z=0.02 Z_{\odot}$ from the code of Maraston (2005), we estimated that the average ionizing photon energy is $E_{v}=21.4 \mathrm{eV}$ and that more than $99 \%$ of the photons have an energy lower than $4 E_{v_{\lim }}$. According to the Gould \& Weinberg (1996) calculation, the fraction of energy of the UV photon that is emitted as $\operatorname{Ly} \alpha$ radiation due to the collisional excitations/relaxations is given by

$$
E_{\mathrm{exc}} / E_{v} \sim 0.08+0.1\left(1-\frac{2 v_{\mathrm{lim}}}{v}\right) \sim 0.1 .
$$

For a cloud with the properties considered here this yields an energy in Ly $\alpha$ per ionizing photon of $E_{\mathrm{exc}} \approx 2.14 \mathrm{eV}$ or $3.43 \times 10^{-12} \mathrm{erg}$. This results in an average of $0.16 \mathrm{Ly} \alpha$ photons per ionizing photon.

Finally, the Ly $\alpha$ luminosity due to excitations in the ISM, $L_{\mathrm{exc}}^{\mathrm{GAL}}$, is then

$$
\begin{aligned}
L_{\mathrm{exc}}^{\mathrm{GAL}}(M, z)= & {\left[1-f_{\mathrm{esc}}(M, z)\right] f_{\mathrm{Ly} \alpha}(z) A_{\mathrm{He}} \dot{N}_{\mathrm{ion}} E_{\mathrm{exc}} } \\
\approx & 4.03 \times 10^{41}\left[1-f_{\mathrm{esc}}(M, z)\right] f_{\mathrm{Ly} \alpha}(z) \\
& \times \frac{\operatorname{SFR}(M, z)}{M_{\odot} \mathrm{yr}^{-1}} \mathrm{erg} \mathrm{s}^{-1}
\end{aligned}
$$

where again it is assumed to be a function of the SFR.

\subsection{Ly $\alpha$ Emission from Gas Cooling}

During the formation of galaxies, gas from the IGM falls into potential wells composed mainly by dark matter that collapsed under its own gravity. The increase in the gas density leads to a high rate of atomic collisions that heat the gas to a high temperature. According to the study of Fardal et al. (2001), most of the gas in potential wells that collapses under its own gravity never reaches its virial temperature, and so a large fraction of the potential energy is released by line emission induced by collisions and excitations from gas with temperatures $T_{K}<2 \times 10^{4} \mathrm{~K}$. At this temperature approximately $50 \%$ of the energy is emitted in Ly $\alpha$ alone.

From Fardal et al. (2001), we can relate the luminosity at the Ly $\alpha$ frequency due to the cooling in galaxies to their baryonic cold mass, $M_{\text {cool }}^{\text {bar }}$, using

$$
\log _{10}\left(L_{\text {cool }}^{\mathrm{GAL}}\right)=1.52 \log _{10}\left(M_{\mathrm{cool}}^{\mathrm{bar}}\right)+26.32,
$$

where both the luminosity and the mass are in solar units. To relate this baryonic cold mass to a quantity we can use in our models, we used the relation between cold baryonic mass and the halo mass from the galaxies in the Guo et al. (2011) catalog. From the equation above, we can then obtain an expression for the luminosity, which can be fitted by

$$
\begin{aligned}
L_{\mathrm{cool}}^{\mathrm{GAL}}(M) \approx & 1.69 \times 10^{35} f_{\mathrm{Ly} \alpha}(z)\left(1+\frac{M}{10^{8}}\right) \\
& \times\left(1+\frac{M}{2 \times 10^{10}}\right)^{2.1}\left(1+\frac{M}{3 \times 10^{11}}\right)^{-3} \mathrm{erg} \mathrm{s}^{-1}
\end{aligned}
$$

with $M$ in units of $M_{\odot}$. The relation between the cold gas mass and the mass of the halo shows very little evolution with redshift during reionization. Thus, we expect the relation in Equation (13) to only depend on redshift due to the redshift evolution of $f_{\mathrm{Ly} \alpha}$.

\subsection{Contributions from Continuum Emission}

Continuum emission can also contribute to the Ly $\alpha$ observations. These include stellar emission, free-free emission, free-bound emission, and two-photon emission. Photons emitted with frequencies close to the Lyn lines should scatter within the ISM and eventually get re-emitted out of the galaxy as Ly $\alpha$ photons. Otherwise, they will escape the ISM before redshifting into one of the Lyn lines and being reabsorbed by a hydrogen atom.

The fraction of photons that scatter in the galaxy can be estimated from the intrinsic width of the Ly $\alpha$ line, which has $\approx 4 \AA$ (Jensen et al. 2013). We calculated the stellar contribution assuming an emission spectrum for stars with a metallicity of $Z_{\star}=0.02 Z_{\odot}$ estimated with the code from Maraston (2005) that can be approximated by the emission of a blackbody with a temperature of $6.0 \times 10^{4} \mathrm{~K}$ for $h v<13.6 \mathrm{eV}$. The number of stellar origin Ly $\alpha$ photons per solar mass in star formation obtained with this method is

$$
\begin{aligned}
Q_{\mathrm{Ly} \alpha}^{\text {stellar }} & =4.307 \int_{v_{\mathrm{L} y \alpha+2 \AA}}^{v_{\mathrm{L} y \alpha-2 \AA}} d v \frac{v^{3}}{e^{h \nu / K_{b} T_{K}}-1} M_{\odot}^{-1} \\
& =9.92 \times 10^{58} M_{\odot}^{-1} .
\end{aligned}
$$

We note that we are not accounting for the higher opacity at the center of the Ly $\alpha$ line, which should push the photons out of the line center before exiting the star, and so we may be overestimating the stellar $\operatorname{Ly} \alpha$ photon emission. 
Free-bound emission and free-free emission are, respectively, originated when free electrons scatter off ions with or without being captured. Following the approach of Fernandez $\&$ Komatsu (2006), the free-free and free-bound continuum luminosity can be obtained using

$$
L_{v}(M, z)=V_{\text {sphere }}(M, z) \varepsilon_{v},
$$

where $V_{\text {sphere }}$ is the volume of the Strömgren sphere, which can be roughly estimated using the ratio between the number of ionizing photons emitted and the number density of recombinations in the ionized volume,

$$
V_{\text {sphere }}(M, z)=\frac{Q_{\text {ion }} \operatorname{SFR}(M, z)\left(1-f_{\text {esc }}\right)}{n_{e} n_{p} \alpha_{\beta}} .
$$

Here $\varepsilon_{v}$ is the total volume emissivity of free-free and free-bound emission, $n_{p}$ is the number density of protons (ionized atoms), and $\alpha_{i}$ is the case $\mathrm{A}$ or case $\mathrm{B}$ recombination coefficient (see Furlanetto et al. 2006).

The volume emissivity estimated by Dopita \& Sutherland (2003) is given by

$$
\varepsilon_{v}=4 \pi n_{e} n_{p} \gamma_{c} \frac{e^{-h v / k T_{K}}}{T_{K}^{1 / 2}} \mathrm{~J} \mathrm{~cm}^{-3} \mathrm{~s}^{-1} \mathrm{~Hz}^{-1},
$$

where $\gamma_{c}$ is the continuum emission coefficient including free-free and free-bound emission given in SI units by

$$
\gamma_{c}=5.44 \times 10^{-46}\left[\bar{g}_{\mathrm{ff}}+\sum_{n=n^{\prime}}^{\infty} \frac{x_{n} e^{x_{n}}}{n} g_{\mathrm{fb}}(n)\right] .
$$

Here $x_{n}=R y /\left(k_{B} T_{K} n^{2}\right)$ ( $k_{B}$ is the Boltzmann constant, $n$ is the level to which the electron recombines, and $R y=$ $13.6 \mathrm{eV}$ is the Rydberg unit of energy) and $\bar{g}_{\mathrm{ff}} \approx 1.1-1.2$ and $g_{\mathrm{fb}}(n) \approx 1.05-1.09$ are the thermally averaged Gaunt factors for free-free and free-bound emission (Karzas \& Latter 1961, values from). The initial level $n^{\prime}$ is determined by the emitted photon frequency and satisfies the condition $c R_{\infty} / n^{\prime 2}<v<$ $c R_{\infty} /\left(n^{\prime}-1\right)^{2}$, where $R_{\infty}=1.1 \times 10^{7} \mathrm{~m}^{-1}$ is the Rydberg constant.

The continuum luminosity per frequency interval $\left(L_{v}\right)$ is related to the $\operatorname{Ly} \alpha$ luminosity emitted from the galaxies by $L_{\text {cont }}=L_{v} \times d v(4 \AA)=f_{\mathrm{Ly} \alpha} Q_{\mathrm{Ly} \alpha} E_{\mathrm{Ly} \alpha} \operatorname{SFR}(M, z)$, where $Q_{\mathrm{Ly} \alpha}$ is the number of emitted Ly $\alpha$ photons per solar mass in star formation. We then obtain $Q_{\mathrm{Ly} \alpha}^{\text {free-free }}=2.13 \times 10^{53} M_{\odot}^{-1}$ for free-free emission and $Q_{\mathrm{Ly} \alpha}^{\text {free-bound }}=2.22 \times 10^{55} M_{\odot}^{-1}$ for free-bound emission.

During recombination there is also the probability of twophoton emission and although these photons have frequencies below the $\operatorname{Ly} \alpha$ frequency there is a small fraction of them of $Q_{\mathrm{Ly} \alpha}^{2-\text { photon }}$ that are emitted so close to the Ly $\alpha$ line, which are included in the Ly $\alpha$ intrinsic width.

The number of Ly $\alpha$ photons that can be originated due to two-photon emission during recombination is given by

$$
Q_{\mathrm{Ly} \alpha}^{2-\text { photon }}=\int_{v_{\mathrm{Ly} \alpha+2 \AA}}^{v_{\mathrm{Ly} \alpha}} \frac{2}{v_{\mathrm{Ly} \alpha}} P\left(\nu / \nu_{\mathrm{Ly} \alpha}\right) d \nu,
$$

where $P(y) d y$ is the normalized probability that in a two-photon decay one of them is the range $d y=d v / \nu_{\text {Ly } \alpha}$ and $1-f_{\text {Ly } \alpha} \approx 1 / 3$ is the probability of two-photon emission during a hydrogen $n=2 \rightarrow 1$ transition. The probability of two-photon decay was fitted by Fernandez \& Komatsu (2006) using Table 4 of Brown \& Mathews (1970) as

$$
\begin{aligned}
P(y)= & 1.307-2.627(y-0.5)^{2}+2.563(y-0.5)^{4} \\
& -51.69(y-0.5)^{6} .
\end{aligned}
$$

Finally, the different contributions to the total $\operatorname{Ly} \alpha$ luminosity from galaxies due to continuum emission, $L_{\text {cont }}^{\mathrm{GAL}}=L_{\mathrm{cont}}^{\text {stellar }}+$ $L_{\text {cont }}^{\text {free-free }}+L_{\text {cont }}^{\text {free-bound }}+L_{\text {cont }}^{2-\text { photon }}$, are given by

$$
\begin{aligned}
L_{\text {cont }}^{\text {stellar }}(M, z) & =f_{\mathrm{Ly} \alpha} Q_{\mathrm{Ly} \alpha}^{\text {stellar }} E_{\mathrm{Ly} \alpha} \operatorname{SFR}(M, z) \\
& \approx 5.12 \times 10^{40} f_{\mathrm{Ly} \alpha} \frac{\operatorname{SFR}(M, z)}{M_{\odot} \mathrm{yr}^{-1}} \mathrm{erg} \mathrm{s}^{-1}
\end{aligned}
$$

for stellar emission,

$$
\begin{aligned}
L_{\text {cont }}^{\text {free-free }}(M, z) & =f_{\mathrm{Ly} \alpha} Q_{\mathrm{Ly} \alpha}^{\text {stellar }} E_{\mathrm{Ly} \alpha} \operatorname{SFR}(M, z) \\
& \approx 1.10 \times 10^{35} f_{\mathrm{Ly} \alpha} \frac{\operatorname{SFR}(M, z)}{M_{\odot} \mathrm{yr}^{-1}} \mathrm{erg} \mathrm{s}^{-1}
\end{aligned}
$$

for free-free emission,

$$
\begin{aligned}
L_{\text {cont }}^{\text {free-bound }}(M, z) & =f_{\text {Ly } \alpha} Q_{\mathrm{Ly} \alpha}^{\text {stellar }} E_{\mathrm{Ly} \alpha} \operatorname{SFR}(M, z) \\
& \approx 1.47 \times 10^{37} f_{\mathrm{Ly} \alpha} \frac{\operatorname{SFR}(M, z)}{M_{\odot} \mathrm{yr}^{-1}} \mathrm{erg} \mathrm{s}^{-1}
\end{aligned}
$$

for free-bound emission, and

$$
\begin{aligned}
L_{\text {cont }}^{2-\text { photon }}(M, z) & =f_{\mathrm{Ly} \alpha} Q_{\mathrm{Ly} \alpha}^{\text {stellar }} E_{\mathrm{Ly} \alpha} \operatorname{SFR}(M, z) \\
& \approx 2.41 \times 10^{38} f_{\mathrm{Ly} \alpha} \frac{\operatorname{SFR}(M, z)}{M_{\odot} \mathrm{yr}^{-1}} \mathrm{erg} \mathrm{s}^{-1}
\end{aligned}
$$

for two-photon emission.

Note that here we are only considering the part of the continuum emission from galaxies that could contribute to the same "Ly $\alpha$ redshift." There will be a continuum emission spectrum with frequencies below the Ly $\alpha$ line from the mechanisms above that will contribute to the same observation from lower redshifts and will generate a "foreground" to the $\operatorname{Ly} \alpha$ signal that needs to be removed. This should be possible due to the smoothness of this background across frequency, in the same manner as foregrounds of the $21 \mathrm{~cm}$ signal are removed (e.g., Wang et al. 2006).

\subsection{Modeling the Relation between Star Formation Rate and Halo Mass}

Simulations of galaxy formation and observations indicate that the star formation of a halo increases strongly for small halo masses, but at high halo masses $\left(M \gtrsim 10^{11} M_{\odot}\right)$ it becomes almost constant (Conroy \& Wechsler 2009; Popesso et al. 2012).

In order to better estimate and constrain the SFR of a halo, we used three nonlinear SFR versus halo mass parameterizations that are in good agreement with different observational constraints. In Sim 1 we adjusted the SFR to reproduce a reasonable reionization history and an $\mathrm{Ly} \alpha \mathrm{LF}$ evolution compatible with different observational constraints, and in $\operatorname{Sim} 2$ we adjusted the SFR versus halo mass relation to the parameterizations from the Guo et al. (2011) galaxy catalog (low halo masses) and the De Lucia \& Blaizot (2007) galaxy catalog (high halo masses). Sim2 results in an early reionization history with an optical depth to 


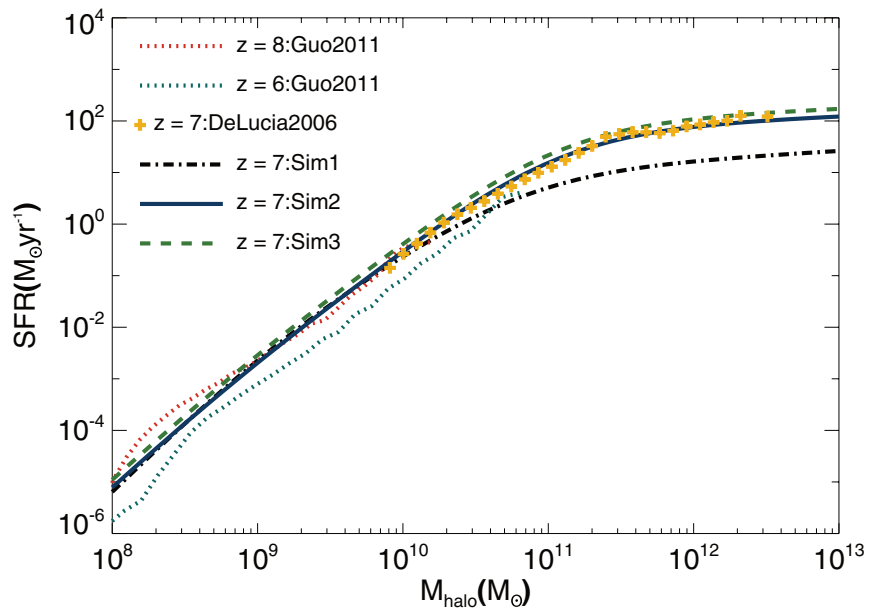

Figure 1. Star formation rate vs. halo mass. The dotted lines show the relations taken from the Guo et al. (2011) catalog for low halo masses at $z=6$ (bottom dotted line) and $z=8$ (upper dotted line); the yellow crosses show the relation taken from the DeLucia catalog for high halo masses at $z=7$. The dash-dotted, solid, and dashed lines show the parameterizations used in simulations Sim1, $\operatorname{Sim} 2$, and $\operatorname{Sim} 3$, respectively, for $z=7$.

(A color version of this figure is available in the online journal.)

reionization compatible with the low bound of the current observational constraints. Finally, Sim 3 has the same halo mass dependence as $\operatorname{Sim} 2$ but evolves with redshift in a similar way to the De Lucia \& Blaizot (2007) and Guo et al. (2011) galaxy catalogs.

We parameterized the relations between the SFR and halo mass as

$$
\frac{\operatorname{SFR}(M, z)}{M_{\odot} / \mathrm{yr}}=\left(2.8 \times 10^{-28}\right) M^{a}\left(1+\frac{M}{c_{1}}\right)^{b}\left(1+\frac{M}{c_{2}}\right)^{d},
$$

where $a=2.8, b=-0.94, d=-1.7, c_{1}=1 \times 10^{9} M_{\odot}$, and $c_{2}=7 \times 10^{10} M_{\odot}$ for $\operatorname{Sim} 1$;

$$
\begin{aligned}
\frac{\operatorname{SFR}(M, z)}{M_{\odot} / \mathrm{yr}}= & 1.6 \times 10^{-26} M^{a}\left(1+\frac{M}{c_{1}}\right)^{b} \\
& \times\left(1+\frac{M}{c_{2}}\right)^{d}\left(1+\frac{M}{c_{3}}\right)^{e},
\end{aligned}
$$

where $a=2.59, b=-0.62, d=0.4, e=-2.25, c_{1}=$ $8 \times 10^{8} M_{\odot}, c_{2}=7 \times 10^{9} M_{\odot}$, and $c_{3}=1 \times 10^{11} M_{\odot}$ for $\operatorname{Sim} 2$; and

$$
\begin{aligned}
\frac{\operatorname{SFR}(M, z)}{M_{\odot} / \mathrm{yr}}= & 2.25 \times 10^{-26}(1+0.075 \times(z-7)) M^{a} \\
& \times\left(1+\frac{M}{c_{1}}\right)^{b}\left(1+\frac{M}{c_{2}}\right)^{d}\left(1+\frac{M}{c_{3}}\right)^{e},
\end{aligned}
$$

where $a=2.59, b=-0.62, d=0.4, e=-2.25, c_{1}=$ $8 \times 10^{8} M_{\odot}, c_{2}=7 \times 10^{9} M_{\odot}$, and $c_{3}=1 \times 10^{11} M_{\odot}$ for $\operatorname{Sim} 3$. Figure 1 shows these relations.

In Figure 2, the strong decline in the observational SFR density (SFRD) from $z \approx 8$ to $z \approx 10$, imposed by the observational point at $z=10.3$, was obtained with the observation of a single galaxy using the Hubble Deep Field 2009 two years' data (Bouwens et al. 2011; Oesch et al. 2012). It was argued in Bouwens et al. (2012a), based on an analytical calculation,

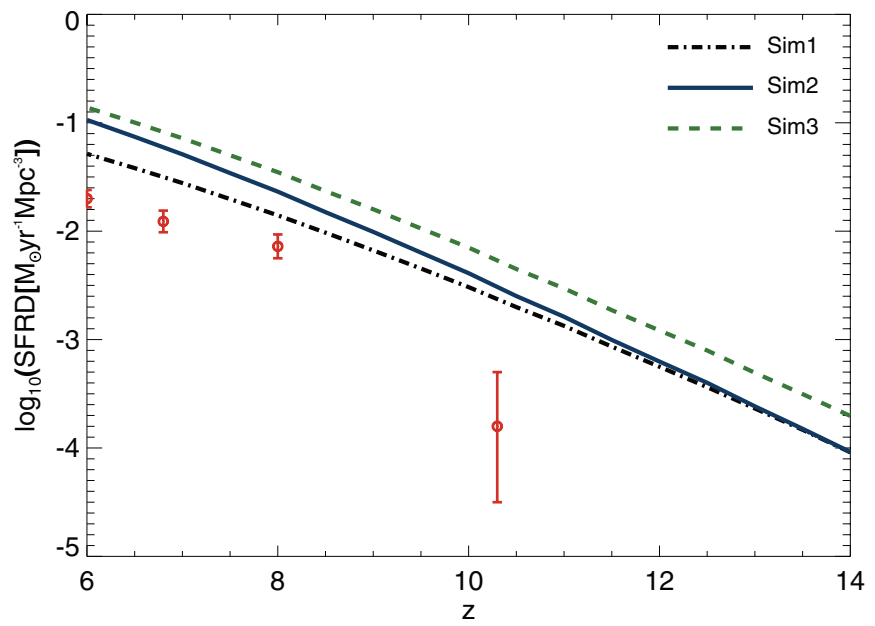

Figure 2. Star formation rate density evolution as a function of redshift. The blue solid line, the green dashed line, and the black dash-dotted line were obtained from simulations made using the SimFast 21 code (for information about the code see Section 4 and Santos et al. 2010) and the SFR vs. halo mass relations from Equations (24)-(26). The red dots are observational constraints derived from the UV luminosities corrected for dust extinction from Bouwens et al. (2012b). Please note that these observational values correspond to highmass galaxies, while our results integrate over the halo-mass function starting at $\sim 10^{8}$ solar masses (which at redshift 7 corresponds to star formation rates of $6.41 \times 10^{-5} M_{\odot} \mathrm{s}^{-1}$ for $\operatorname{Sim} 1,7.83 \times 10^{-6} M_{\odot} \mathrm{s}^{-1}$ for $\operatorname{Sim} 2$, and $1.1 \times 10^{-5} M_{\odot} \mathrm{s}^{-1}$ for Sim3), so our star formation rate densities are expected to be higher.

(A color version of this figure is available in the online journal.)

that even with such low SFRD at high redshifts it was possible to obtain an optical depth to reionization compatible with the value obtained by Wilkinson Microwave Anisotropy Probe (WMAP; $\tau=0.088 \pm 0.015$ ) (Komatsu et al. 2011). However, this derivation would imply a high escape fraction of ionizing radiation, and that reionization would end at $z \approx 8$, which is hard to reconcile with the constraints from observations of quasar spectra (Mesinger \& Haiman 2007; Zaldarriaga et al. 2008). Our SFRDs are considerably higher than the current observational constraints, although the difference can be explained by a systematic underestimation of the SFR in observed galaxies. Moreover, current observations only probe the high-mass end of the high-redshift galaxies' mass function, which will underestimate the SFRD (also the obtained SFRs have very high error bars due to uncertainties in the correction due to dust extinction, the redshift, and the galaxy type). In the following sections the results shown were obtained using Siml unless stated otherwise.

\subsection{Total Lya Luminosity: Comparison with Observations}

In the previous sections we calculated the Ly $\alpha$ luminosity as a function of the SFR for several effects. The commonly used "empirical" relation between these two quantities is (Jiang et al. 2011)

$$
L_{\mathrm{Gal}}=1.1 \times 10^{42} \frac{\mathrm{SFR}(M, z)}{M_{\odot} \mathrm{yr}^{-1}} \mathrm{erg} \mathrm{s}^{-1},
$$

and it is based on the relation between SFR and the $\mathrm{H} \alpha$ luminosity from Kennicutt (1998a) and on the line emission ratio of $\operatorname{Ly} \alpha$ to $\mathrm{H} \alpha$ in case $\mathrm{B}$ recombinations calculated assuming a gas temperature of $10^{4} \mathrm{~K}$. This empirical relation gives the Ly $\alpha$ luminosity without dust absorption (we have labeled it $K 98$ for the remainder of the paper).

Our relation between luminosity and star formation is mass dependent (both from the escape fraction and due to the 
Table 2

Average Luminosity per Star Formation Rate (in Units of erg s $\mathrm{s}^{-1} / M_{\odot} \mathrm{yr}^{-1}$ ) Averaged over the Halo Mass Function for Redshifts 10, 9, 8, and 7 , from Top to Bottom

\begin{tabular}{lcccc}
\hline \hline$A_{\text {rec }}(z)$ & $A_{\text {exc }}(z)$ & $A_{\text {cool }}(z)$ & $A_{\text {cont }}(z)$ & $A_{\text {total }}(z)$ \\
\hline $4.4 \times 10^{41}$ & $1.1 \times 10^{41}$ & $1.3 \times 10^{39}$ & $8.1 \times 10^{40}$ & $6.4 \times 10^{41}$ \\
$1.2 \times 10^{42}$ & $3.2 \times 10^{41}$ & $7.8 \times 10^{38}$ & $6.4 \times 10^{40}$ & $1.6 \times 10^{42}$ \\
$9.3 \times 10^{41}$ & $2.4 \times 10^{41}$ & $4.9 \times 10^{38}$ & $4.9 \times 10^{40}$ & $1.2 \times 10^{42}$ \\
$8.5 \times 10^{41}$ & $2.2 \times 10^{41}$ & $3.3 \times 10^{38}$ & $3.6 \times 10^{40}$ & $1.1 \times 10^{42}$ \\
\hline
\end{tabular}

expression from the cooling mechanism), so in order to compare it with the result above, we calculate

$$
A(z)=\frac{\left\langle L_{\mathrm{Gal}}(M, z)\right\rangle}{\langle\operatorname{SFR}(M, z)\rangle},
$$

where the average $\langle x\rangle$ of quantity $x$ is done over the halo mass function for the mass range considered. The results are presented in Table 2 for a few redshifts.

Although our Ly $\alpha$ luminosities per SFR are slightly higher, at least for low redshifts, we point out that the "empirical" relation is based on a theoretical calculation that only accounts for Ly $\alpha$ emission due to recombinations. Moreover, the observational measurements of $\mathrm{H} \alpha$ and $\mathrm{Ly} \alpha$ are primarily made at low redshifts, where the absorption of Ly $\alpha$ photons by dust in galaxies is expected to be high. Our relation has the advantage of evolving with redshift since it accounts for the evolution of the escape fraction of ionizing photons and for the evolution of the escape fraction of Ly $\alpha$ photons. This $z$-dependence is not present in the standard empirical relation. This redshift evolution of the UV photons' escape fraction is a consequence of the increase in the number of massive galaxies with more clumpy structure as the redshift decreases. The star-forming regions of massive galaxies are embedded in clumps, and therefore it becomes more difficult for the ionizing photons to escape from such dense regions (Razoumov \& Sommer-Larsen 2010; Yajima et al. 2011). The redshift evolution of the relation presented in Equation (28) justifies why a theoretical calibration between Ly $\alpha$ luminosity and the SFR of a galaxy is useful for our work.

To check the consistency between our theoretical estimation of the Ly $\alpha$ luminosity and the existing observations during reionization, we show in Figure 3 the LF using two of the SFR versus halo mass parameterizations presented in Section 2.5. This prediction is then compared to Ly $\alpha$ LFs of photometric identified objects in Shimasaku et al. (2006) and in Kashikawa et al. (2006) near the end of the reionization epoch.

Our LFs were calculated assuming a minimum halo mass of $8 \times 10^{8} M_{\odot}$, which corresponds to a minimum luminosity of $3.72 \times 10^{36} \mathrm{erg} \mathrm{s}^{-1}$ for Sim1, $4.49 \times 10^{36} \mathrm{erg} \mathrm{s}^{-1}$ for Sim2, and $6.22 \times 10^{36} \mathrm{erg} \mathrm{s}^{-1}$ for Sim3. The agreement between our LFs and observations is reasonable for Sim 1; however, our Sim 2 overpredicts the abundance of high-luminosity $\operatorname{Ly} \alpha$ emitters. This difference can be due to sample variance or a result of the high sensitivity of theoretical predictions to several parameters in our model. We point out that the luminosity range relevant for this comparison falls in a halo mass range outside the one for which the escape fraction of UV radiation we are using was estimated, so we could easily get a better fit between observations and $\operatorname{Sim} 2$ by reducing this escape fraction for high halo masses. This difference could also be related with the choice of halo mass function. Here we choose the Sheth-Tormen halo mass function (Sheth \& Tormen 1999), which has been shown to fit low-redshift simulations more accurately, but it

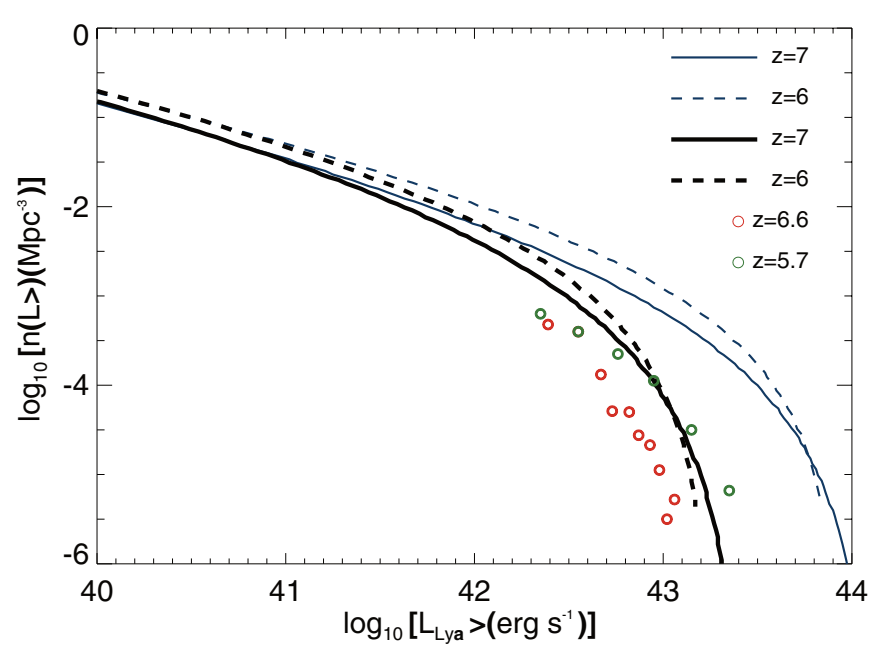

Figure 3. Ly $\alpha$ luminosity functions obtained with our calculations are shown for redshifts $z=6$ (dashed lines) and $z=7$ (solid lines) for Siml (black thick lines) and $\operatorname{Sim} 2$ (blue thin lines). The green and red circles show the intrinsic (i.e., not affected by the IGM) Ly $\alpha$ LF from photometric identified objects in Shimasaku et al. (2006) and in Kashikawa et al. (2006) for $z=5.7$ and 6.6, respectively.

(A color version of this figure is available in the online journal.)

is yet to be established the extent to which such a halo mass function can reproduce the halo distribution during reionization. Another possible explanation for this difference is the existence of a small amount of neutral gas in the IGM, which would severely decrease the observed Ly $\alpha$ luminosity from galaxies. Also, we could have decreased the high-luminosity end of our LFs if we had use an Ly $\alpha$ escape fraction that decreased with halo mass such as the one used in Forero-Romero et al. (2011). We do not consider a model fit to the data to optimize various parameters in our model given that the current constraints on the observed Ly $\alpha$ LFs have large overall uncertainties, especially considering variations from one survey to another.

\subsection{Lya Average Intensity}

In this section and the next one we will attempt to estimate the intensity and power spectrum of the Ly $\alpha$ signal using an analytical model. In Section 4, we will improve the estimation by doing the same calculation using a semi-numerical simulation.

The total intensity of Ly $\alpha$ emission can be obtained from the combined luminosity of Ly $\alpha$ photons associated with different mechanisms described in the previous sub-sections, such that

$$
\bar{I}_{\mathrm{Gal}}(z)=\int_{M_{\min }}^{M_{\max }} d M \frac{d n}{d M} \frac{L_{\mathrm{Gal}}(M, z)}{4 \pi D_{\mathrm{L}}^{2}} y(z) D_{\mathrm{A}}^{2},
$$

where $d n / d M$ is the halo mass function (Sheth \& Tormen 1999), $M$ is the halo mass, $M_{\max }=10^{13} M_{\odot}, M_{\min }=M_{\mathrm{OB}}, D_{\mathrm{L}}$ is the proper luminosity distance, and $D_{\mathrm{A}}$ is the comoving angular diameter distance. Finally, $y(z)=d \chi / d v=\lambda_{\mathrm{Ly} \alpha}(1+z)^{2} / H(z)$, where $\chi$ is the comoving distance, $v$ is the observed frequency, and $\lambda_{\text {Ly } \alpha}=2.46 \times 10^{-15} \mathrm{~m}$ is the rest-frame wavelength of the Ly $\alpha$ line.

The evolution of the Ly $\alpha$ intensity predicted by this calculation is shown in Figure 4 together with the scaling expected under the "empirical" relation from Kennicutt (1998a) combined with an assumption related to the gas temperature. The intensities of Ly $\alpha$ emission from different sources are presented in Table 3 for several redshifts. 


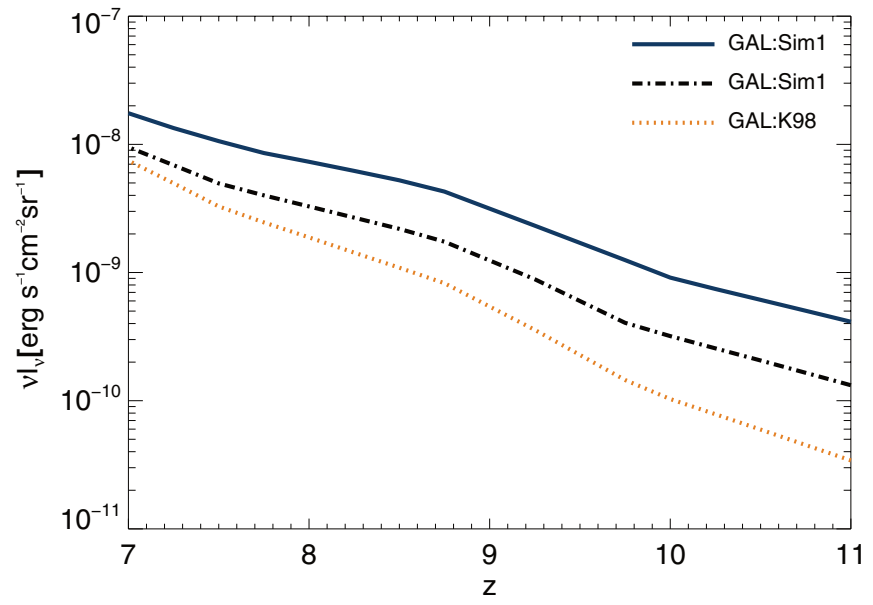

Figure 4. Ly $\alpha$ intensity from galaxies in $\mathrm{erg} \mathrm{s}^{-1} \mathrm{~cm}^{-2} \mathrm{sr}^{-1}$ as a function of redshift. The black dash-dotted line and the blue solid line were obtained using our theoretical calculation of the Ly $\alpha$ luminosity and the SFR halo mass relation from Sim1 and Sim2, respectively. The orange dotted line uses the Ly $\alpha$ luminosity SFR relation based on the relation between SFR and the H $\alpha$ luminosity from Kennicutt (1998a) and the line emission ratio of Ly $\alpha$ to $\mathrm{H} \alpha$ in case $\mathrm{B}$ recombinations calculated assuming a gas temperature of $10,000 \mathrm{~K}$ (labeled as the K98 relation). The K98 line is not corrected for dust absorption. (A color version of this figure is available in the online journal.)

Table 3

Surface Brightness (in Observed Frequency times Intensity) of Ly $\alpha$ Emission from the Different Sources in Galaxies at $z \approx 7, z \approx 8$, and $z \approx 10$ for Sim 1

\begin{tabular}{llll}
\hline \hline $\begin{array}{l}\text { Source of emission in } \\
\left(\mathrm{erg} \mathrm{s}^{-1} \mathrm{~cm}^{-2} \mathrm{sr}^{-1}\right)\end{array}$ & $v I_{v}(z=7)$ & $v I_{v}(z=8)$ & $v I_{v}(z=10)$ \\
\hline Recombinations & $7.3 \times 10^{-9}$ & $2.5 \times 10^{-9}$ & $2.3 \times 10^{-10}$ \\
Excitations & $1.9 \times 10^{-9}$ & $6.5 \times 10^{-10}$ & $6.0 \times 10^{-11}$ \\
Cooling & $2.8 \times 10^{-12}$ & $1.5 \times 10^{-12}$ & $4.7 \times 10^{-13}$ \\
Continuum & $3.1 \times 10^{-10}$ & $3.5 \times 10^{-10}$ & $3.0 \times 10^{-11}$ \\
$\quad$ Total & $9.5 \times 10^{-9}$ & $3.5 \times 10^{-9}$ & $3.2 \times 10^{-10}$ \\
\hline
\end{tabular}

These intensities can be extrapolated to other SFRDs, assuming that the only change is in the amplitude of the SFR halo mass relations presented in Figure 1 by using the coefficients in Table 4.

The intensities from emission at $z \approx 7,8$, and 10 are $9.5 \times 10^{-9}, 3.5 \times 10^{-9}$, and $3.2 \times 10^{-10} \mathrm{erg} \mathrm{s}^{-1} \mathrm{~cm}^{-2} \mathrm{sr}^{-1}$, respectively. Such an intensity is substantially smaller than the background intensity of integrated emission from all galaxies (around $1 \times 10^{-5} \mathrm{erg} \mathrm{s}^{-1} \mathrm{~cm}^{-2} \mathrm{sr}^{-1}$; Madau \& Pozzetti 2000), or from the total emission of galaxies during reionization, estimated to be at most $1 \times 10^{-6} \mathrm{erg} \mathrm{s}^{-1} \mathrm{~cm}^{-2} \mathrm{sr}^{-1}$ (Cooray et al. 2012).

\subsection{Ly $\alpha$ Intensity Power Spectrum}

The Ly $\alpha$ emission from galaxies will naturally trace the underlying cosmic matter density field, so we can write the Ly $\alpha$ line intensity fluctuations due to galaxy clustering as

$$
\delta I_{\mathrm{GAL}}=b_{\mathrm{Ly} \alpha} \bar{I}_{\mathrm{GAL}} \delta(\mathbf{x})
$$

where $\bar{I}_{\mathrm{GAL}}$ is the mean intensity of the Ly $\alpha$ emission line, $\delta(\mathbf{x})$ is the matter overdensity at the location $\mathbf{x}$, and $b_{\mathrm{Ly} \alpha}$ is the average galaxy bias weighted by the Ly $\alpha$ luminosity (see, e.g., Gong et al. 2011).

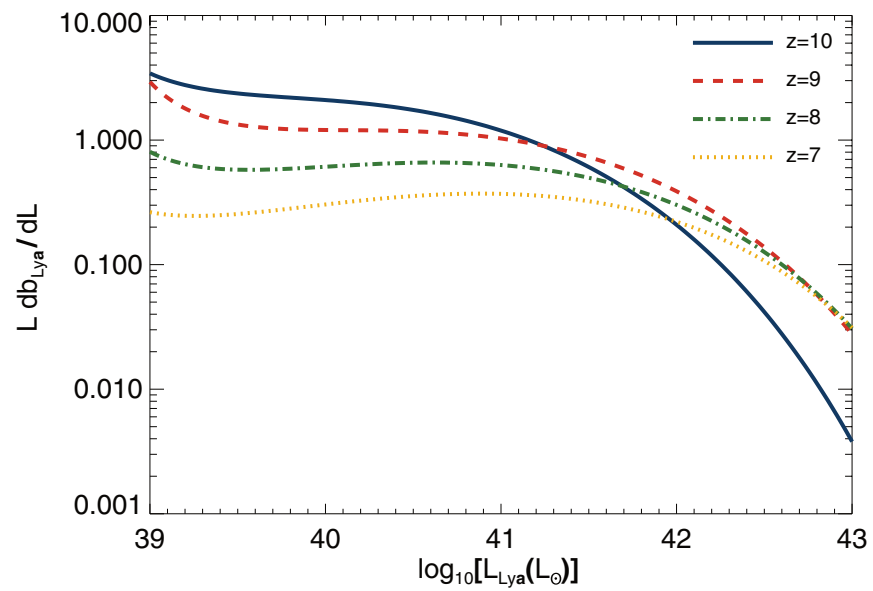

Figure 5. Bias between dark matter fluctuations and Ly $\alpha$ surface brightness $(v I)$ from galaxies as a function of the galaxy Ly $\alpha$ luminosity at redshifts 7, 8, 9 , and 10 .

(A color version of this figure is available in the online journal.)

Table 4

Average Ly $\alpha$ Intensity from Galaxies per SFRD ( $\left.A_{\mathrm{SFRD}}\right)$ in Units of erg s${ }^{-1} \mathrm{~cm}^{-2} \mathrm{sr}^{-1} / M_{\odot} \mathrm{yr}^{-1}$, Calculated Using the Star Formation Rate Halo Mass Relation from Simulations Sim1 and Sim2

\begin{tabular}{lll}
\hline \hline Redshift & $A_{\text {SFRD }}(\operatorname{Sim} 1)$ & $A_{\text {SFRD }}(\operatorname{Sim} 2)$ \\
\hline 10 & $1.05 \times 10^{-03}$ & $2.20 \times 10^{-03}$ \\
9 & $2.17 \times 10^{-03}$ & $3.75 \times 10^{-03}$ \\
8 & $2.34 \times 10^{-03}$ & $3.15 \times 10^{-03}$ \\
7 & $3.42 \times 10^{-03}$ & $3.42 \times 10^{-03}$ \\
\hline
\end{tabular}

Using one of the relations between the SFR and halo mass from Section 2.5, we can calculate the luminosity and obtain the Ly $\alpha$ bias following Visbal \& Loeb (2010):

$$
b_{\mathrm{Ly} \alpha}(z)=\frac{\int_{M_{\min }}^{M_{\max }} d M \frac{d n}{d M} L_{\mathrm{GAL}} b(z, M)}{\int_{M_{\min }}^{M_{\max }} d M \frac{d n}{d M} L_{\mathrm{GAL}}},
$$

where $b(z, M)$ is the halo bias and $d n / d M$ is the halo mass function (Sheth \& Tormen 1999). We take $M_{\min }=10^{8} M_{\odot} / h$ and $M_{\max }=10^{13} M_{\odot} / h$. The bias between dark matter fluctuation and the Ly $\alpha$ luminosity, as can be seen in Figure 5, is dominated by the galaxies with low $\operatorname{Ly} \alpha$ luminosity independently of the redshift.

We can then obtain the clustering power spectrum of Ly $\alpha$ emission as

$$
P_{\mathrm{GAL}}^{\text {clus }}(z, k)=b_{\mathrm{Ly} \alpha}^{2} \bar{I}_{\mathrm{GAL}}^{2} P_{\delta \delta}(z, k),
$$

where $P_{\delta \delta}(z, k)$ is the matter power spectrum. The shot-noise power spectrum, due to discretization of the galaxies, is also considered here. It can be written as (Gong et al. 2011)

$$
P_{\mathrm{Ly} \alpha}^{\mathrm{shot}}(z)=\int_{\mathrm{M}_{\max }}^{\mathrm{M}_{\min }} d M \frac{d n}{d M}\left(\frac{L_{\mathrm{GAL}}}{4 \pi D_{\mathrm{L}}^{2}} y(z) D_{\mathrm{A}}^{2}\right)^{2} .
$$

The resulting power spectrum of $\operatorname{Ly} \alpha$ emission from galaxies is presented in Figure 6. At all scales presented the Ly $\alpha$ intensity and fluctuations are dominated by the recombination emission from galaxies.

\section{Ly $\alpha$ EMISSION FROM THE IGM}

The Ly $\alpha$ emission from the IGM is mostly originated in recombinations and collisions powered by the ionizing 

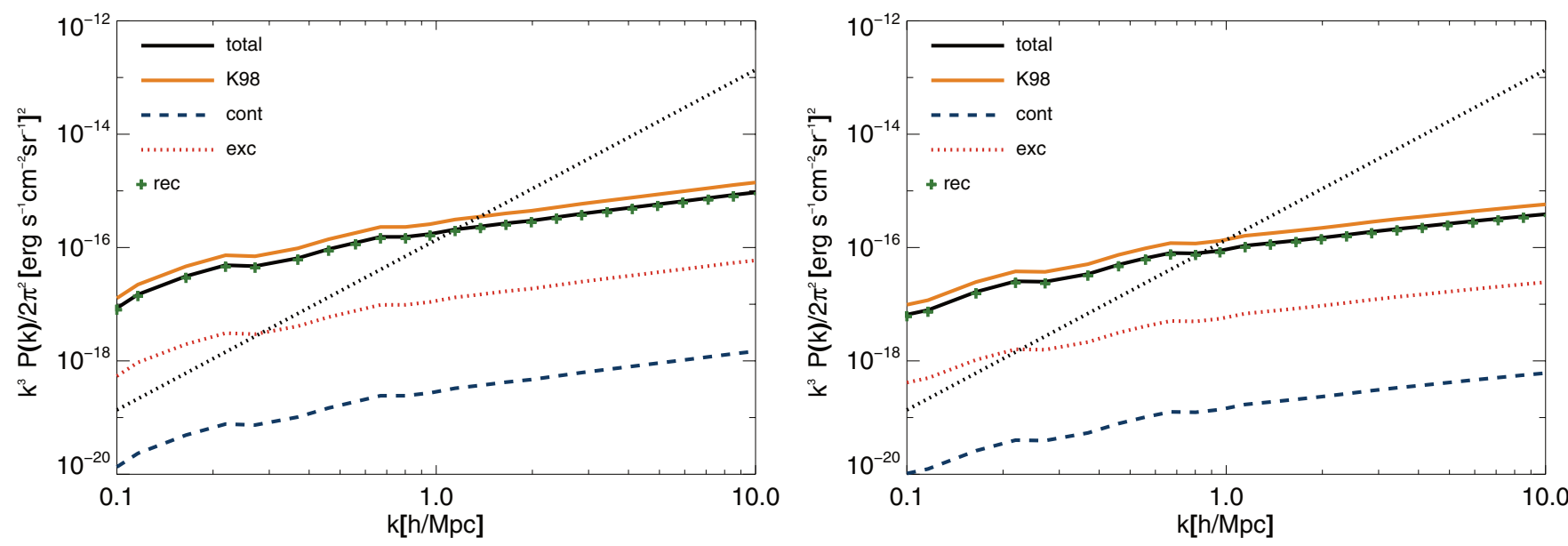

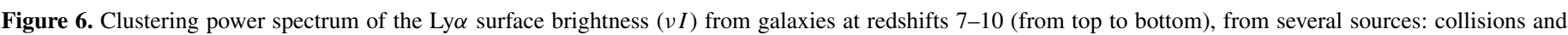

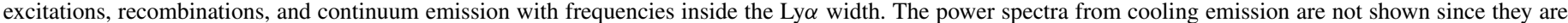

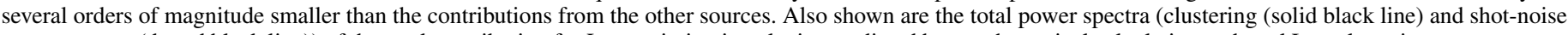

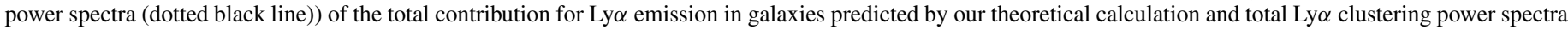
predicted using the $K 98$ relation (orange solid line).

(A color version of this figure is available in the online journal.)

background. These processes are similar to the ones described inside the galaxies, although, since the physical conditions of the gas in the IGM are different from those in the ISM, the intensity of Ly $\alpha$ emission can no longer be connected to the ionizing photon intensity using the previous relations. The biggest challenge in doing these calculations is to connect the IGM ionizations and heating of the gas to the emission of ionizing radiation and the SFR assumed in the previous sections. Moreover, in the IGM, we also have to take into account the contribution of continuum radiation from stars between the Ly $\alpha$ and the Lyman limit, which redshifts into the $\operatorname{Ly} \alpha$ line.

In a schematic view, we have to take into account the following processes:

1. The amount of energy in UV photons that escapes the galaxy.

2. This energy will then be distributed in the IGM into
(a) ionizations,
(b) direct excitations (followed by emission, partially into the Ly $\alpha$ line), and
(c) heating of the gas.

3. Taking into account the state of the IGM in terms of temperature and ionization, we can then further determine how much it will radiate through the Ly $\alpha$ line from
(a) recombinations and
(b) radiative cooling (usually through excitations followed by decay in several lines including $\operatorname{Ly} \alpha$ ).

4. The amount of Lyn photons that escape the galaxy, rescattering in the IGM into Ly $\alpha$ photons.

The proper calculation of all these processes will require simulations, which we will address in Section 4. In the following sub-sections we review the contributions through analytical calculations in order to get a better understanding of the dominating effects.

\subsection{Lya Emission from Hydrogen Recombinations}

The UV radiation that escapes the ISM into the IGM ionizes low-density clouds of neutral gas. Part of the gas in these clouds then recombines, giving rise to Ly $\alpha$ emission. The radiation emitted in the IGM is often referred to as fluorescence (Santos 2004). The comoving number density of recombinations per second in a given region, $\dot{n}_{\text {rec }}$, is given by

$$
\dot{n}_{\mathrm{rec}}(z)=\alpha n_{e}(z) n_{\mathrm{H} \text { II }}(z),
$$

where $\alpha$ changes between the case $\mathrm{A}$ and the case $\mathrm{B}$ recombination coefficient and $n_{\mathrm{H} \text { II }}=x_{i}\left(n_{b}\left(1-Y_{p}\right) /\left((1-3) / 4 Y_{p}\right)\right)$ is the ionized hydrogen comoving number density ( $x_{i}$ is the ionization fraction, $n_{b}$ the baryonic comoving number density). The free electron density can be approximated by $n_{e}=x_{i} n_{b}$.

The recombination coefficients are a function of the IGM temperature, $T_{K}$. The case A comoving recombination coefficient is appropriate for the highly ionized low-redshift universe (Furlanetto et al. 2006),

$$
\alpha_{A} \approx 4.2 \times 10^{-13}\left(T_{K} / 10^{4} \mathrm{~K}\right)^{-0.7}(1+z)^{3} \mathrm{~cm}^{3} \mathrm{~s}^{-1},
$$

while the case B comoving recombination coefficient is appropriate for the high-redshift universe,

$$
\alpha_{B} \approx 2.6 \times 10^{-13}\left(T_{K} / 10^{4} \mathrm{~K}\right)^{-0.7}(1+z)^{3} \mathrm{~cm}^{3} \mathrm{~s}^{-1} .
$$

The use of a larger recombination coefficient when the process of hydrogen recombination is close to its end accounts for the fact that, at this time, ionizations (and hence recombinations) take place in dense, partially neutral gas (Lyman-limit systems) and the photons produced after recombinations are consumed inside these systems, so they do not help ionize the IGM (see, e.g., Furlanetto et al. 2006).

The fraction of Ly $\alpha$ photons emitted per hydrogen recombination, $f_{\text {rec }}$, is temperature dependent, so we used the parameterization for $f_{\text {rec }}$ made by Cantalupo et al. (2008) using a combination of fits tabulated by Pengelly (1964) and Martin (1988) for $T_{K}>10^{3}$ and $T_{K}<10^{3}$, respectively:

$$
f_{\text {rec }}=0.686-0.106 \log _{10}\left(T_{K} / 10^{4} \mathrm{~K}\right)-0.009\left(T_{K} / 10^{4} \mathrm{~K}\right)^{-0.4} \text {. }
$$

The luminosity density (per comoving volume) in Ly $\alpha$ from hydrogen recombinations in the IGM, $\ell_{\mathrm{rec}}^{\mathrm{IGM}}$, is then given by

$$
\ell_{\mathrm{rec}}^{\mathrm{IGM}}(z)=f_{\mathrm{rec}} \dot{n}_{\mathrm{rec}} E_{\mathrm{Ly} \alpha}
$$




\subsection{Lya Emission from Excitations in the IGM}

The UV radiation that escapes the galaxies without producing ionization ends up ionizing and exciting the neutral hydrogen in the IGM and heating the gas around the galaxies. The high energetic electron released after the first ionization spends its energy in collisions/excitations, ionizations, and heating the IGM gas until it thermalizes (Shull \& van Steenberg 1985). We estimated the contribution of the direct collisions/excitations to the Ly $\alpha$ photon budget and concluded that it is negligible.

The Ly $\alpha$ luminosity density due to the collisional emission (radiative cooling in the IGM), $\ell_{\mathrm{exc}}^{\mathrm{IGM}}$, is given by

$$
\ell_{\mathrm{exc}}^{\mathrm{IGM}}(z)=n_{e} n_{\mathrm{HI}} q_{\mathrm{Ly} \alpha} E_{\mathrm{Ly} \alpha},
$$

where $n_{\mathrm{H}}=n_{b}\left(1-x_{i}\right)\left(\left(1-Y_{p}\right) /\left((1-3) / 4 Y_{p}\right)\right)$ is the neutral hydrogen density, $x_{i}$ is the IGM ionized fraction, and $q_{\mathrm{Ly} \alpha}$ is the effective collisional excitation coefficient for Ly $\alpha$ emission, which we calculated in the same way as Cantalupo et al. (2008), but using different values for the gas temperature and IGM ionization fraction.

Considering excitation processes up to the level $n=3$ that could eventually produce Ly $\alpha$ emission, the effective collisional excitation coefficient is given by

$$
q_{\mathrm{Ly} \alpha}=q_{1,2 p}+q_{1,2 s}+q_{1,3 p} .
$$

The collisional excitation coefficient for the transition from the ground level (1) to the level $(n l)$ is given by

$$
q_{1, n l}=\frac{8.629 \times 10^{-6}}{T_{K}^{1 / 2}} \frac{\Omega(1, n l)}{\omega_{1}} e^{E_{1, n} / k_{B} T_{K}} \mathrm{~cm}^{3} \mathrm{~s}^{-1},
$$

where $\Omega(1, n l)$ is the temperature-dependent effective collision strength, $\omega_{1}$ is the statistical weight of the ground state, $E_{1, n}$ is the energy difference between the ground and the $n l$ level, and $k_{B}$ is the Boltzmann constant.

\subsection{Scattering of Lyn Photons Emitted from Galaxies}

Continuum emission of photons, by stars, from Ly $\alpha$ to the Lyman limit travels until it reaches one of the Lyn lines, where it gets scattered by neutral hydrogen. Most of this scattering will have as an end result the production of Ly $\alpha$ photons, which eventually redshift out of the line. Since a considerable fraction of these photons only reach a given Lyn frequency in the IGM, this Ly $\alpha$ emission is formed as a flux that decays with $r^{2}$ around the star that emitted the continuum photons, so it appears diluted in frequency in line observations of point sources (Chen $\&$ Miralda-Escudé 2008). These continuum photons are much less likely to be absorbed by the dust in the ISM than photons originated in recombinations.

In intensity mapping, the frequency band observed is much larger than in line observations, so in principle all the continuum Ly $\alpha$ photons can be detected. Using the spectral energy distribution (SED) made with the code from Maraston (2005), we estimated that the number of photons emitted by stars between the $\operatorname{Ly} \alpha$ plus the Ly $\alpha$ equivalent width and the Lyman limit is equivalent to $Q_{\mathrm{Lyn}}^{\mathrm{IGM}}=9.31 \times 10^{60} M_{\odot}^{-1} \mathrm{~s}^{-1}$. The higher frequency photons are absorbed by hydrogen atoms as they reach the $\operatorname{Ly} \beta$ frequency, re-emitted, and suffer multiple scattering until they reach the $\operatorname{Ly} \alpha$ line. The fraction of the continuum photons emitted close to the Ly $\alpha$ line have already redshifted to lower frequencies before reaching the IGM, so they will not

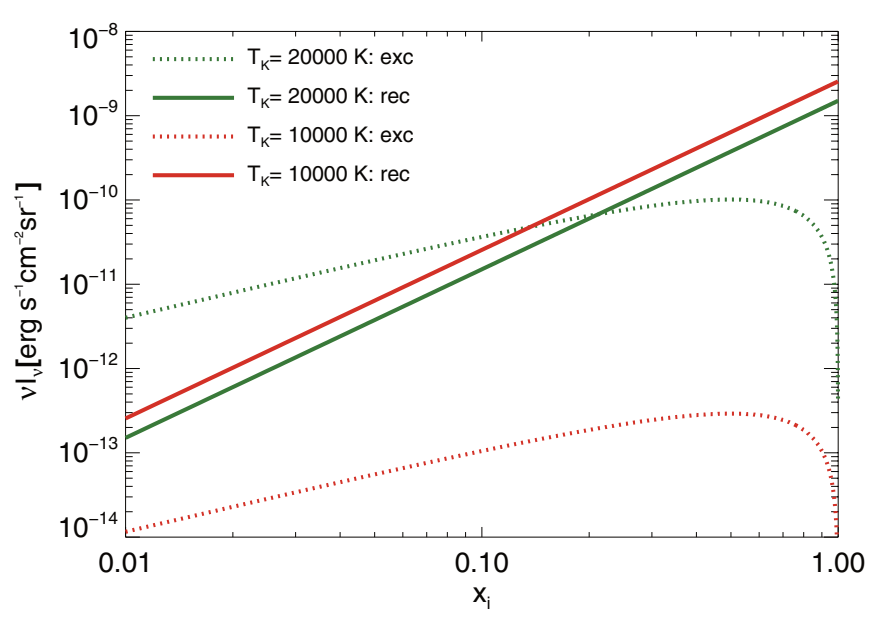

Figure 7. Intensity of $\mathrm{Ly} \alpha$ emission at redshift 7 due to recombinations and excitations in the IGM as a function of the hydrogen ionized fraction. The green and red lines assume a constant gas temperature of $20,000 \mathrm{~K}$ and $10,000 \mathrm{~K}$, respectively.

(A color version of this figure is available in the online journal.)

be scattered by the neutral hydrogen in the IGM and will not contribute to the radiative coupling of the $21 \mathrm{~cm}$ signal (they are already included in the calculation of the Ly $\alpha$ emission from galaxies).

The intensity of this emission was calculated with a stellar emissivity that evolves with frequency as $v^{-\alpha}$ with $\alpha=0.86$ and normalized to $Q_{\mathrm{Lyn}}^{\mathrm{IGM}}$. The Ly $\alpha$ luminosity density originated from continuum stellar radiation and emitted in the IGM, $\ell_{\text {cont }}^{\text {IGM }}$, is then approximately given by

$$
\ell_{\text {cont }}^{\mathrm{IGM}}(z) \approx Q_{\mathrm{ly} \alpha}^{\mathrm{IGM}} E_{\mathrm{Ly} \alpha} \operatorname{SFRD}(z),
$$

where the SFRD is in units of $M_{\odot} \mathrm{s}^{-1}$. Note that in Section 4, this calculation is done through a full simulation.

\subsection{Ly $\alpha$ Intensity}

We calculated the intensities for the several Ly $\alpha$ sources in the IGM from their luminosity densities using

$$
\bar{I}_{\mathrm{IGM}}(z)=\frac{\ell^{\mathrm{IGM}}(z)}{4 \pi D_{L}^{2}} y(z) D_{A}^{2} .
$$

The luminosity and hence the intensity of $\operatorname{Ly} \alpha$ emission in the IGM depend on local values of the hydrogen ionized fraction, the gas temperature, and the gas density. These parameters are correlated with each other, and so theoretical calculations of the average intensity made with the average of these parameters may be misleading. Since this emission is dominated by overdense regions, a clumping factor of a few units is usually assumed in theoretical calculations. However, we decided to estimate this intensity without using a clumping factor since its effect can be extrapolated from the intensity without clumping. The intensity of $\operatorname{Ly} \alpha$ emission due to recombinations or collisions in the IGM is shown in Figure 7 as a function of the hydrogen ionized fraction for different values of the gas temperature.

Even for a fixed average IGM ionized fraction, the intensity of $\operatorname{Ly} \alpha$ emission is the result of emission from several regions, and so all the values shown in Figure 7 are relevant. As can be seen in Figure 7, the intensity of Ly $\alpha$ due to recombinations and collisions in the IGM is very sensitive to the gas temperature and to the fluctuations in the IGM ionized fraction. 


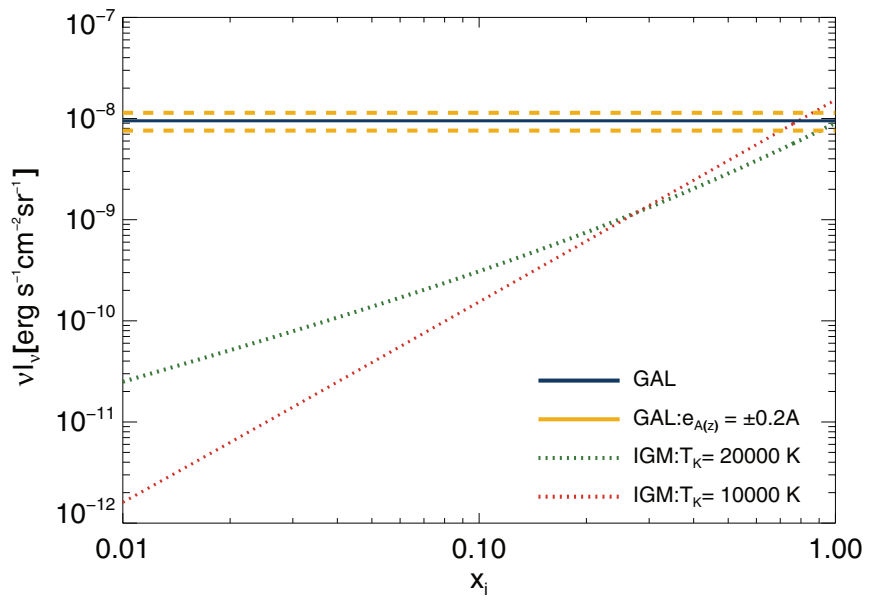

Figure 8. Intensity of Ly $\alpha$ emission at redshift 7 from the IGM and from galaxies as a function of the hydrogen ionized fraction and including all contributions. The green and red lines are the intensity of Ly $\alpha$ emission in the IGM assuming a constant gas temperature of $20,000 \mathrm{~K}$ and $10,000 \mathrm{~K}$, respectively. The blue solid line is the intensity of $\mathrm{Ly} \alpha$ emission from galaxies as calculated in the previous section. The yellow dashed lines show the intensity in galaxies assuming an error in $A(z)$ of $20 \%$ due to the uncertainty in the ionizing photons' escape fraction and due to the uncertainty in the emissivity of ionizing photons. The intensities in the IGM were calculated assuming a clumping factor of 6 compatible with current conservative estimates (Pawlik et al. 2010).

(A color version of this figure is available in the online journal.)

Numerical simulations predict that the temperatures in the hydrogen gas in the IGM can vary from 5000 to 20,000 K (Davé et al. 2001; Smith et al. 2011). The theoretical intensities of Ly $\alpha$ emission in galaxies and in the IGM shown in Figure 8 indicate that unless the IGM clumping factor is very high, or the Ly $\alpha$ photon escape fraction is very low, the $\operatorname{Ly} \alpha$ intensity from the IGM at $z=7$ is lower than the emission from galaxies. At higher redshifts the SFRD will decrease, causing the Ly $\alpha$ intensities from galaxies and from the IGM to decrease. The escape fraction of UV photons from galaxies increases as the redshift increases, which will contribute negatively to the intensity of emission in galaxies and positively to the intensity of emission in the IGM. At high redshifts the IGM ionized fraction is small, which contributes to a strong decrease in the intensity of emission from the IGM compared to the intensity at $z=7$.

\section{Ly $\alpha$ INTENSITY AND POWER SPECTRUM USING NUMERICAL SIMULATIONS}

The intensity of Ly $\alpha$ emission in the IGM at a given time and a given region is proportional to the ionized fraction, the gas temperature, and the matter density in that region. Since these three quantities are correlated, the use of average values in the Ly $\alpha$ intensity calculation highly underestimates the emission in the more overdense regions. Also the evolution of the average of the IGM ionized fraction is poorly known during the EoR.

Some of these problems can be resolved using a computational code able to produce simulations of the IGM ionized fraction, the gas temperature, and the matter density in a volume high enough to properly represent our universe. The use of simulations has an additional advantage of allowing the calculation of the three-dimensional power spectra of $\operatorname{Ly} \alpha$ emission in the IGM without the need for assuming a bias relation with the underlying dark matter distribution.

In this section we will estimate the inhomogeneous Ly $\alpha$ intensity from galaxies and the IGM using a modified version of the SimFast 21 code (Santos et al. 2010). Given a set of astrophysical and cosmological parameters, this code is able to consistently produce three-dimensional simulations of the dark matter density field, the ionization field, the SFRD, the scattering of Lyn photons in the IGM, the X-ray heating of the IGM, and even $21 \mathrm{~cm}$ spin and brightness temperature fluctuations for the several redshifts of the EoR.

A proper calculation of all the heating and cooling mechanisms would add a high level of complexity to this calculation and would require a small redshift step in the IGM fraction calculation, so we assumed a constant temperature in ionized regions of 10,000 K. Moreover, the results from our calculations can be easily extrapolated to account for a higher temperature. For example, for a temperature of $20,000 \mathrm{~K}$ the number of recombinations in the IGM would decrease by a factor of 1.7 and the number of collisions would increase more than two orders in magnitude. Assuming that the clumping of the IGM is not very high, and so Ly $\alpha$ recombination emission dominates over collisional emission during most of the EoR, this higher temperature would cause a small decrease in the intensity of emission in the IGM and the reionization period would be less extended than what we predict in Section 4.1. We made a few modifications to the SimFast 21 code in order to provide a consistent description of the ionization history and its relations to the $\mathrm{Ly} \alpha$ emission, which we now describe.

\subsection{IGM Ionized Fraction Calculation}

In the previous version of the SimFast21 code, the IGM ionized fraction was computed assuming that at each redshift the ionization state of a region could be estimated from the collapsed mass in that region assuming a linear relation between collapsed mass and ionizing power. So a given spherical region of radius $R$ is considered ionized if (Furlanetto et al. 2006)

$$
\zeta M_{\text {coll }}(R) \geqslant M_{\text {tot }}(R)
$$

where $M_{\text {coll }}$ is the collapsed mass that corresponds to the total mass in halos in that region, $M_{\text {tot }}$ is the total mass in the region, and $\zeta$ is an ionizing efficiency parameter. This efficiency parameter tries to include all the ionizations and recombinations produced by a halo as a function of its mass but has no actual physical meaning, although its use is somewhat justified by the large uncertainty in the astrophysical quantities involved in the determination of the relation between halo mass and ionizing efficiency and in the adjustment of this parameter in order to reproduce a reionization history compatible with observations.

In order to calculate the Ly $\alpha$ field, however, we need to include the recombinations in the IGM explicitly, as well as directly relate the ionization process to the emitted stellar radiation. We therefore modified the SimFast 21 code to include these improvements. This new method allows a nonlinear relation between collapsed mass and ionizing power, and all the parameters involved in the calculation have values based on current astrophysical constraints. Also, the size of ionized regions is now set by the volume at which the total ionizing emissivity of the sources it contains equals the number of recombinations so that the system is in equilibrium. For each redshift the implementation of this method was done with the following steps:

1. A halo catalog with the mass and three-dimensional spatial positions was generated using the same method used in the original version of the SimFast 21 code.

2. We calculated SFRs from the halo catalogs using the nonlinear relations in Equations (24)-(26). 


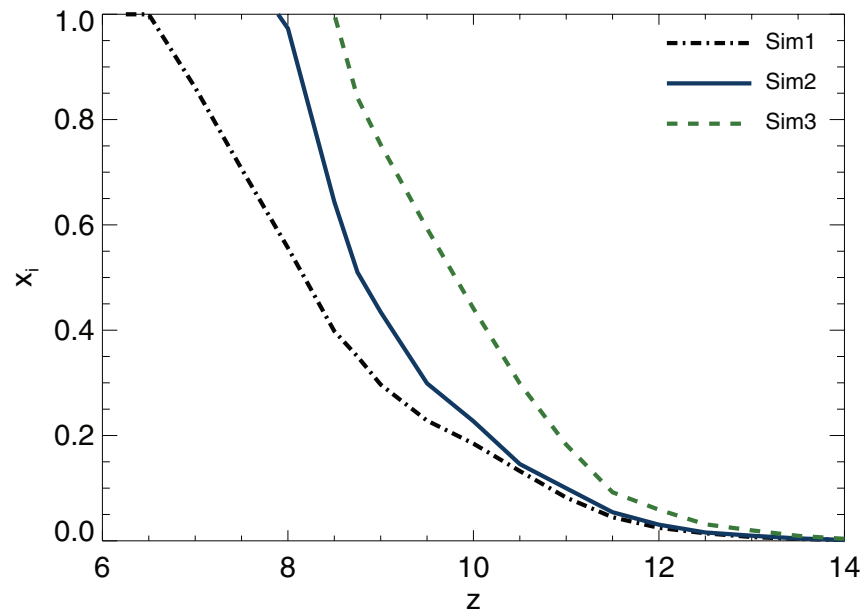

Figure 9. Evolution of the IGM ionized fraction as a function of redshift for the three star formation rate halo mass parameterizations shown in Section 2.5.

(A color version of this figure is available in the online journal.)

3. We used Equation (4) to obtain the halo ionizing rate, $\dot{N}_{\text {ion }}$, and we corrected for the presence of helium using $A_{\mathrm{He}}$ and multiplied it by $f_{\text {esc }}$, to account for the photons consumed inside the galaxies:

$$
\dot{N}_{\text {ion }}^{\text {IGM }}(z, M)=A_{\mathrm{He}} \dot{N}_{\text {ion }}(M) f_{\text {esc }}(z, M) .
$$

The UV ionizing rates of the halos, $\dot{N}_{\text {ion }}^{\text {IGM }}$, were then put in three-dimensional boxes.

4. Three-dimensional boxes with the rate of recombinations in each cell, $\dot{N}_{\text {rec }}^{\mathrm{IGM}}=V_{\text {cell }} \times \dot{n}_{\text {rec }}^{\mathrm{IGM}}$, were obtained from a dark matter density simulation made with the SimFast21 code using Equation (34) with $x_{i}$ set to 1 and $T_{K}=10^{4} \mathrm{~K}$.

5. Following the same procedure as in the original version of the SimFast 21 code, we applied a series of top-hat filters of decreasing size (this filtering procedure was done in Fourier space) to the ionizing rate and the recombination rate boxes in order to calculate the region ionizing rate and recombination rate.

6. At each filtering step of radius $R$ we found the ionized regions ( $\mathrm{H}$ II bubbles) by checking if the region ionizing rate was equal to or higher than its recombination rate. With this method $\mathrm{H}$ II bubbles are always fully ionized:

$$
\dot{N}_{\text {ion }}^{\mathrm{IGM}}(z, R) \geqslant \dot{N}_{\text {rec }}^{\mathrm{IGM}} \text {. }
$$

\subsection{Intensity from Recombinations and Collisions in the IGM}

The SimFast21 code was built to calculate the IGM ionized state assuming two types of regions: one fully ionized (inside the $\mathrm{H}$ II bubbles) and the other fully neutral. The intensity of Ly $\alpha$ emission in the IGM due to recombinations is a smooth function of the IGM ionized fraction and is dominated by emission from fully ionized regions (see Figure 7), so the output of the SimFast 21 code is good enough to estimate this intensity.

Collisions between electrons and neutral hydrogen atoms can also lead to Ly $\alpha$ emission; however, as was explained in Section 3.2, collisional Ly $\alpha$ emission only occurs in partly ionized regions, mainly in the edge of $\mathrm{H}$ II bubbles, so the estimation of this emission requires a more detailed description of the IGM ionized state than the one given by the limited resolution of semi-numerical simulations. Collisions are most important in regions where the IGM ionized fraction is locally close to 0.5
Table 5

Surface Brightness (in Observed Frequency times Intensity) of Ly $\alpha$ Emission from the Different Sources in the IGM at $z \approx 7, z \approx 8$, and $z \approx 10$

\begin{tabular}{llll}
\hline \hline $\begin{array}{l}\text { Source of emission in } \\
\left(\mathrm{erg} \mathrm{s}^{-1} \mathrm{~cm}^{-2} \mathrm{sr}^{-1}\right)\end{array}$ & $v I_{v}(z=7)$ & $v I_{v}(z=8)$ & $v I_{v}(z=10)$ \\
\hline Recombinations & $9.3 \times 10^{-10}$ & $4.8 \times 10^{-10}$ & $9.6 \times 10^{-11}$ \\
Continuum & $3.5 \times 10^{-13}$ & $1.2 \times 10^{-13}$ & $1.5 \times 10^{-14}$ \\
$\quad$ Total & $1.6 \times 10^{-9}$ & $6.7 \times 10^{-10}$ & $1.1 \times 10^{-10}$ \\
\hline
\end{tabular}

and the temperatures are high. Since high-temperature regions are likely to be highly ionized, we can deduce with the help of Figure 7 that Ly $\alpha$ emission from recombinations is dominant over Ly $\alpha$ emission from collisions in the IGM.

\subsection{Intensity from the Scattering of Lyn Photons in the IGM}

The IGM Ly $\alpha$ intensity from scattering of Lyn photons emitted from galaxies can also be calculated using data from the code SimFast21. This code uses Equation (10) in Santos et al. (2010) to calculate the spherical average of the number of Ly $\alpha$ photons, $J_{\alpha}$, hitting a gas element per unit proper area per unit time per unit frequency per steradian. The Ly $\alpha$ intensity originated from these continuum photons is given by

$$
I_{\mathrm{cont}}^{\mathrm{IGM}}=\frac{6 J_{\alpha} E_{\mathrm{Ly} \alpha} D_{\mathrm{A}}^{2}}{(1+z)^{2} D_{\mathrm{L}}^{2}} .
$$

\subsection{Results}

Using the prescriptions described in the previous sections, we ran simulations $\operatorname{Sim} 1, \operatorname{Sim} 2$, and $\operatorname{Sim} 3$ with a volume of $54^{3} h^{-3} \mathrm{Mpc}^{3}$ and 1800 cells from redshift 14 to redshift 6. The obtained IGM ionization fractions, at redshift 7, where $x_{i}=0.86$ for simulation Sim 1 and $x_{i}=1.0$ for simulations Sim2 and Sim3. These values are consistent with the current most likely values for this parameter, $0.8 \leqslant x_{i}(z=7) \leqslant 1.0$ (Mitra et al. 2012). The IGM ionized fraction evolution for Sim 2 and for Sim3 (see Figure 9) resulted in optical depths to reionization of 0.073 and 0.082 . These optical depths are consistent with the value obtained by WMAP ( $\tau=0.088 \pm 0.015$; Komatsu et al. 2011). The optical depth corresponding to Siml is 0.66, which is lower than the current observational constraints. Based on the optical depth constraint, $\operatorname{Sim} 2$ and $\operatorname{Sim} 3$ have the most likely reionization histories, and the IGM ionized fraction evolution obtained with Siml can be seen as a lower bound.

The intensities of $\mathrm{Ly} \alpha$ emission from galaxies at redshift 7 obtained with the SimFast 21 code are similar to the more theoretical estimates summarized in Table 3.

Intensities of $\operatorname{Ly} \alpha$ emission in the IGM made with the same code are presented in Table 5.

The intensity values found in Tables 3 and 5 and the theoretical estimations plotted in Figure 8 indicate that for the Ly $\alpha$ intensity from the IGM to reach a value close to the emission from galaxies at $z=7$ would require a very large absorption of Ly $\alpha$ photons by dust in galaxies.

The resulting power spectra of $\mathrm{Ly} \alpha$ emission in galaxies and in the IGM obtained with the SimFast 21 code are presented in Figure 10 for $z=7$ and $z=10$.

We repeated the Ly $\alpha$ power spectra calculation for several redshifts during the EoR and plotted the Ly $\alpha$ power spectra as a function of redshift for several $k$ in Figure 11.

We calculated the intensity of Ly $\alpha$ emission from galaxies and from the IGM (intensities are shown in Figure 12) and found 

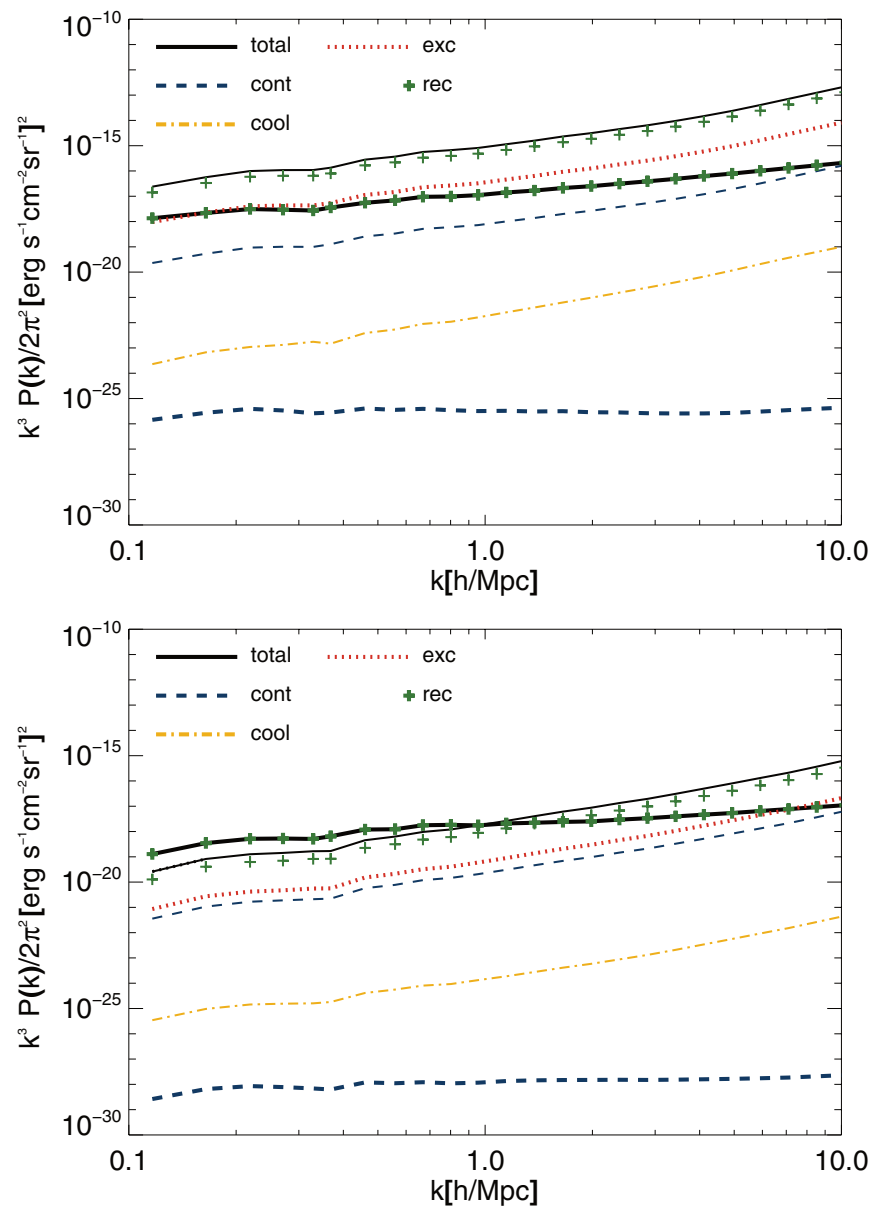

Figure 10. Power spectrum of Ly $\alpha$ surface brightness $(v I)$ from galaxies (thin lines) and from the IGM (thick lines) at redshifts 7 (top) and 10 (bottom). The shown contributions to the Ly $\alpha$ flux are from excitations and collisions, recombinations, continuum emission inside the Ly $\alpha$ width (from galaxies), scattering of Lyn photons (from the IGM), cooling emission in galaxies, and the total emission.

(A color version of this figure is available in the online journal.)

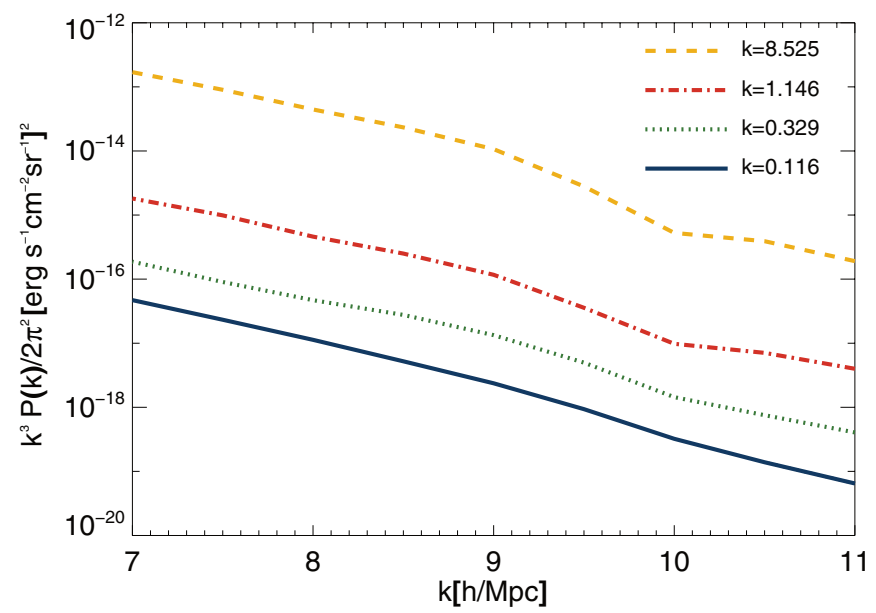

Figure 11. Total power spectrum of Ly $\alpha$ emission during the EoR as a function of redshift.

(A color version of this figure is available in the online journal.)

that, according to our assumptions and as already previously seen, the Ly $\alpha$ emission from galaxies is dominant over the Ly $\alpha$ emission from the IGM at least during the redshift interval from $z=6$ to $z=9$.

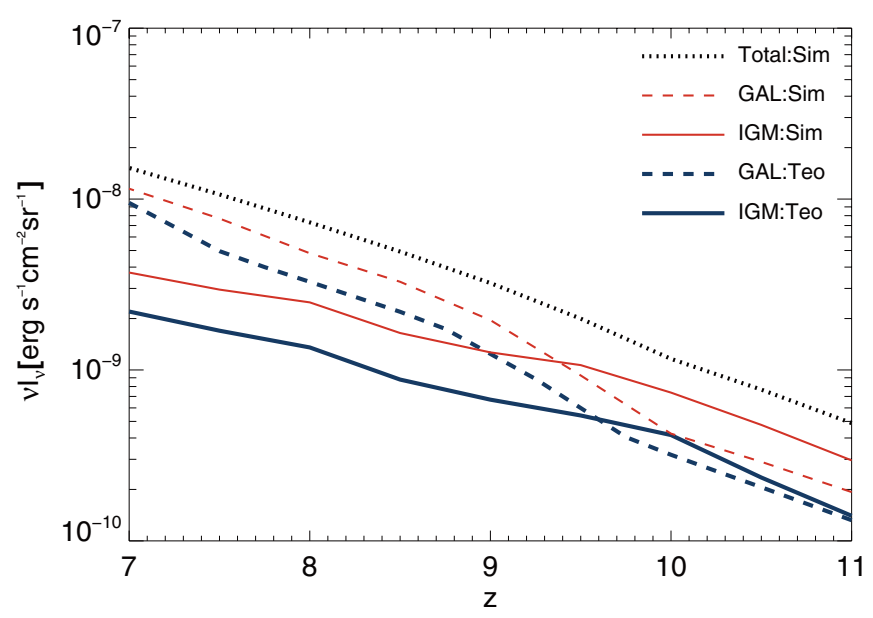

Figure 12. Ly $\alpha$ intensity from galaxies (dashed lines), from the IGM (solid lines) as a function of redshift from our simulation (red thin lines), and from the theoretical calculations (blue thick lines). Also shown is the total Ly $\alpha$ emission from the simulation (dotted line). All the intensities were calculated using the star formation halo mass relation from Sim 1. The theoretical intensity of Ly $\alpha$ emission from the IGM was calculated using the average IGM ionization values obtained from Sim1.

(A color version of this figure is available in the online journal.)

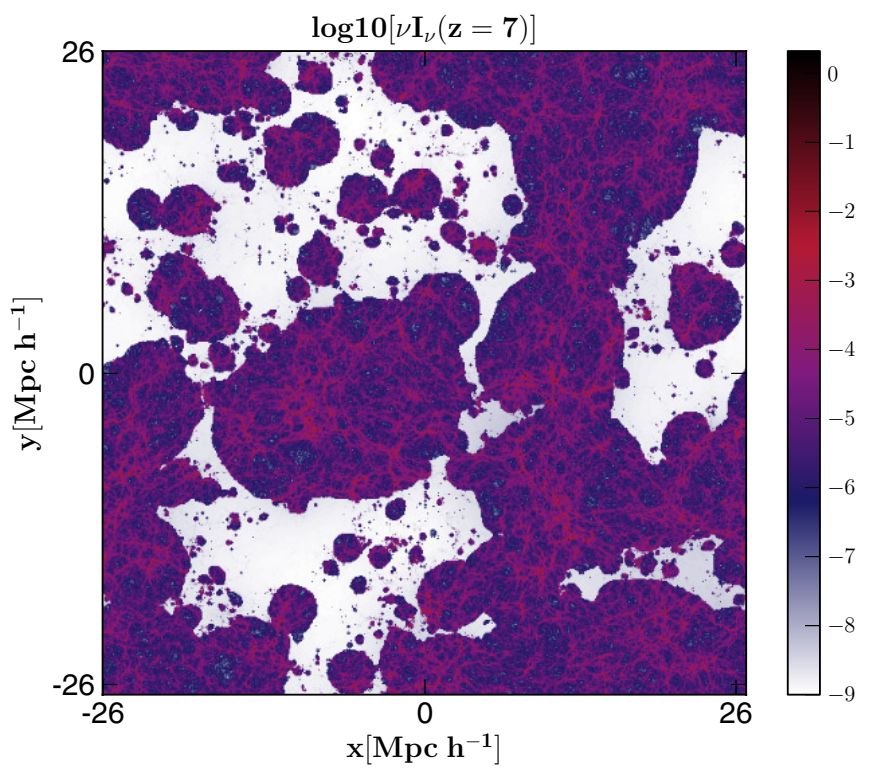

Figure 13. Total Ly $\alpha$ intensity from galaxies and the IGM in erg s $\mathrm{sm}^{-1} \mathrm{sr}^{-1}$ at redshift 7.

(A color version of this figure is available in the online journal.)

Also, a map of the total Ly $\alpha$ intensity in galaxies and in the IGM is presented in Figure 13 for $z=7$.

Since the star formation halo mass relation is not very constrained, we can use the results obtained with Sim 1 and Sim3 as the lower and upper bounds to the expected Ly $\alpha$ intensity. The evolution of the Ly $\alpha$ intensity from galaxies, from the IGM, and from galaxies plus IGM can be seen, respectively, in Tables 6, 7, and 8 for simulations $\operatorname{Sim} 1, \operatorname{Sim} 2$, and $\operatorname{Sim} 3$.

\section{CROSS-CORRELATION BETWEEN Ly $\alpha$ AND $21 \mathrm{~cm}$ OBSERVATIONS}

Observations of the $21 \mathrm{~cm}$ signal from the EoR will suffer from contamination by foregrounds and systematic effects. Since both $21 \mathrm{~cm}$ line emission and Ly $\alpha$ line emission trace 
Table 6

Surface Brightness (in Observed Frequency times Intensity) in Units of $\mathrm{erg} \mathrm{s}^{-1} \mathrm{~cm}^{-2} \mathrm{sr}^{-1}$ of Ly $\alpha$ Emission from Galaxies at $z \approx 7, z \approx 8$, and $z \approx 10$ for $\operatorname{Sim} 1, \operatorname{Sim} 2$, and $\operatorname{Sim} 3$

\begin{tabular}{lccc}
\hline \hline Simulation & $v I_{v}(z=7)$ & $v I_{v}(z=8)$ & $v I_{v}(z=10)$ \\
\hline Sim1 & $1.43 \times 10^{-8}$ & $5.13 \times 10^{-9}$ & $4.55 \times 10^{-11}$ \\
Sim2 & $2.54 \times 10^{-8}$ & $8.34 \times 10^{-9}$ & $5.68 \times 10^{-11}$ \\
Sim3 & $3.57 \times 10^{-8}$ & $1.26 \times 10^{-8}$ & $9.73 \times 10^{-11}$ \\
\hline
\end{tabular}

Table 7

Surface Brightness (in Observed Frequency times Intensity) in Units of $\mathrm{erg} \mathrm{s}^{-1} \mathrm{~cm}^{-2} \mathrm{sr}^{-1}$ of Ly $\alpha$ Emission from the IGM at $z \approx 7, z \approx 8$, and $z \approx 10$ for Sim1, Sim2, and Sim3

\begin{tabular}{lccc}
\hline \hline Simulation & $v I_{v}(z=7)$ & $v I_{v}(z=8)$ & $v I_{v}(z=10)$ \\
\hline Sim1 & $4.33 \times 10^{-9}$ & $3.76 \times 10^{-9}$ & $1.74 \times 10^{-9}$ \\
Sim2 & $6.07 \times 10^{-9}$ & $5.17 \times 10^{-9}$ & $2.18 \times 10^{-9}$ \\
Sim3 & $8.53 \times 10^{-9}$ & $7.81 \times 10^{-9}$ & $3.76 \times 10^{-9}$ \\
\hline
\end{tabular}

Table 8

Surface Brightness (in Observed Frequency times Intensity) in Units of $\mathrm{erg} \mathrm{s}^{-1} \mathrm{~cm}^{-2} \mathrm{sr}^{-1}$ of Total Ly $\alpha$ Emission at $z \approx 7, z \approx 8$, and $z \approx 10$ for $\operatorname{Sim} 1, \operatorname{Sim} 2$, and $\operatorname{Sim} 3$

\begin{tabular}{lccc}
\hline \hline Simulation & $v I_{v}(z=7)$ & $v I_{v}(z=8)$ & $v I_{v}(z=10)$ \\
\hline Sim1 & $1.86 \times 10^{-8}$ & $8.89 \times 10^{-9}$ & $1.79 \times 10^{-9}$ \\
Sim2 & $3.15 \times 10^{-8}$ & $1.35 \times 10^{-8}$ & $2.24 \times 10^{-9}$ \\
Sim3 & $4.42 \times 10^{-8}$ & $2.04 \times 10^{-8}$ & $3.86 \times 10^{-9}$ \\
\hline
\end{tabular}

neutral hydrogen, these two lines are expected to be strongly correlated. The cross-correlation of these two lines can be used as an extra method to probe the evolution of the IGM ionized hydrogen fraction. In particular, the power spectra of this crosscorrelation will have a discontinuity at a scale that is related to the average bubble size and hence the average ionization fraction in the universe.

During the EoR, the $21 \mathrm{~cm}$ signal from galaxies is much smaller than the emission from the IGM, so it is safe to neglect both this galaxy emission and the shot-noise emission in the cross-correlation. Since the Ly $\alpha$ emission from galaxies is dominating over the IGM for most redshifts, we can just concentrate on the Ly $\alpha$-galaxy $/ 21 \mathrm{~cm}$ IGM cross-correlation when analyzing the cross-power spectrum. The cross-correlation between the $21 \mathrm{~cm}$ signal and the Ly $\alpha$ line in galaxies is therefore given by

$$
P_{\mathrm{Ly} \alpha, 21}(z, k)=I_{\mathrm{GAL}} I_{21}\left[P_{\delta \delta}-\frac{1}{1-\bar{x}_{i}} P_{x_{i} \delta}\right],
$$

where $I_{21}$ is the average intensity of $21 \mathrm{~cm}$ emission, $P_{x_{i} \delta}(z, k)$ is the cross-correlation power spectra between the ionized field and the matter density fluctuations, $P_{\delta \delta}(z, k)$ is the power spectra of matter density fluctuations, and we are assuming that the Ly $\alpha$ emission is a biased tracer of the underlying dark matter field.

In Figure 14, we show the cross-correlation power spectrum between the total Ly $\alpha$ emission and the $21 \mathrm{~cm}$ signal at redshifts 7, 8, 9, and 10. For simulation Sim1, these redshifts correspond to ionizing fractions of $x_{i}=0.86,0.56,0.35$, and 0.23 for redshifts 7, 8, 9, and 10, respectively. In Figure 14, the scale at which $P_{\mathrm{Ly} \alpha, 21}(k)$ goes from negative to positive is determined by the average size of the ionized regions. For small scales, the correlation is positive since fluctuations from both lines should be proportional to the underlying density fluctuations, but for large scales (small $k$ ), the correlation is negative since the $21 \mathrm{~cm}$

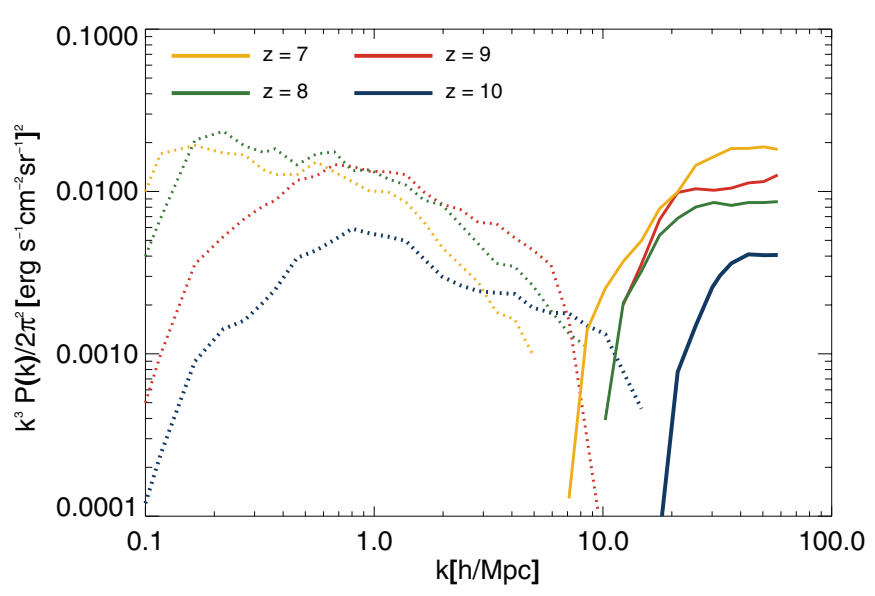

Figure 14. Cross-correlation power spectrum between Ly $\alpha$ emission and the $21 \mathrm{~cm}$ signal for redshifts 7, 8, 9, and 10. Dotted lines indicate a negative correlation, and solid lines indicate a positive correlation.

(A color version of this figure is available in the online journal.)

line and the Ly $\alpha$ line are characteristic of neutral gas and ionized gas, respectively (and there will be an extra negative contribution from the ionized bubbles).

\section{OBSERVATIONS}

Current observations related to $\operatorname{Ly} \alpha$ emission are restricted to narrowband imaging of $\operatorname{Ly} \alpha$ emitters during reionization and the direct detection of individual emitters. This has led to order $\sim 200$ secure detections at $z>5$, but systematic uncertainties remain on the fraction that are arising at low redshifts and associated with $[\mathrm{O} \mathrm{III}] /[\mathrm{O} \mathrm{II}]$ lines, among others. Due to narrow atmospheric windows, observations in near-IR bands necessary to cover the reionization epoch are also limited to multiple discrete bands. In any case, existing data could be used for a statistical study such as the power spectrum to extract properties of Ly $\alpha$ emitters that remain below the $5 \sigma$ level of individual source/line identifications. Given that detections do exist at the bright end and our predictions are consistent with the Ly $\alpha$ LFs derived from observational measurements, it is likely that a modest improvement in existing technology and programs will lead to an experiment with sufficient sensitivity to measure the Ly $\alpha$ anisotropy power spectrum during reionization over a broad range of redshifts. The main limitation, unfortunately, is that existing ground-based observations are very limited to small fields of view with narrow bands in the redshift.

Note that from the ground we expect a noise $(v I)$ of $\sim 2.5 \times 10^{-3} \mathrm{erg} \mathrm{cm}^{-2} \mathrm{sr}^{-1}$ (assuming we can avoid the $\mathrm{OH}$ lines; otherwise, the intensity will be $\sim 1.0 \times 10^{-1} \mathrm{erg} \mathrm{cm}^{-2} \mathrm{sr}^{-1}$ ). From space, the main contamination will be the zodiacal light, which will have a value $\sim 5 \times 10^{-4} \mathrm{erg} \mathrm{cm}^{-2} \mathrm{sr}^{-1}$. It is possible that a dedicated experiment from the ground can be conceived to improve our understanding of reionization through detailed Ly $\alpha$ mapping over a broad range of redshifts using specific instruments and filters that suppress the atmospheric contamination. Because of this strong atmospheric contamination, sub-orbital and/or orbital experiments may, however, offer a better option. The predictions we have made here can be used as a guide in designing such instruments and experiments.

In Figure 15, we show the expected errors at $z=7$ (central wavelength of $0.975 \mu \mathrm{m}$ ) for a dedicated compact space-borne template instrument to study Ly $\alpha$ EoR fluctuations. We consider a $20 \mathrm{~cm}$ aperture and a spectrometer with resolution 


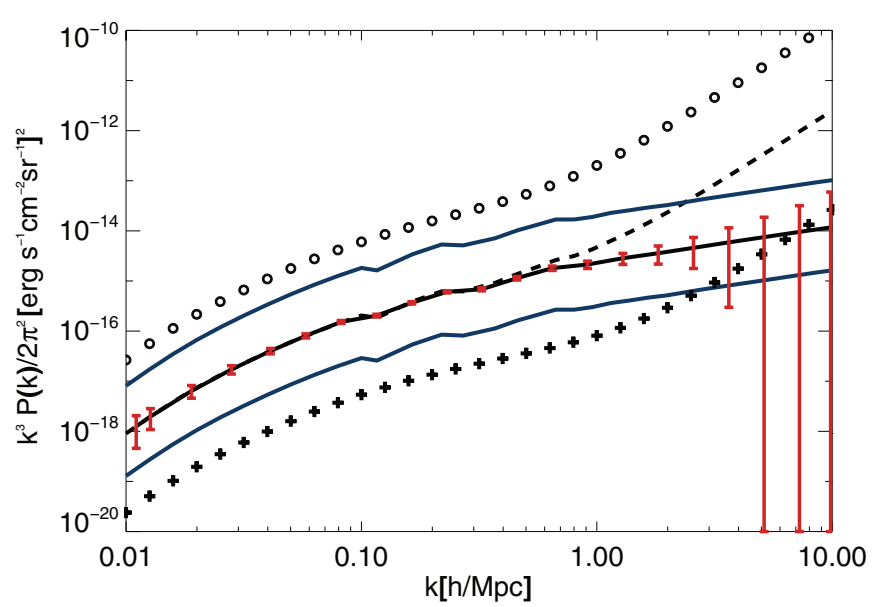

Figure 15. Expected error on the Ly $\alpha$ clustering power spectrum at $z=7$ using a space-based experiment. Black solid line shows the clustering power spectrum for Sim3, while the dashed line includes the shot noise. Red vertical bars show the error. The lower blue solid line shows the clustering power spectrum for Sim1, while the top blue solid line shows the same for a model similar to Sim3 with the same reionization history and optical depth (from WMAP) but with an SFR three times larger and a UV escape fraction three times lower, which will generate an Ly $\alpha$ luminosity function larger than what is usually expected at $z=7$. Circles show the expected $\mathrm{H} \alpha$ power spectrum from $z=0.5$ that will contaminate the observation, and the crosses give the expected $\mathrm{H} \alpha$ signal after galaxies with an $\mathrm{H} \alpha$ luminosity $>10^{40} L_{\odot}$ are removed.

(A color version of this figure is available in the online journal.)

$R=\lambda / \Delta \lambda=200$. The imaging will be done using a $2048 \times$ $2048 \mathrm{HgCdTe}$ detector array in order to cover in one pointing a field of view of $45 \times 45 \mathrm{arcmin}$ with a resolution of $10 \mathrm{arcsec}$ pixels on the sky and a spectral range from 0.85 to $1.10 \mu \mathrm{m}$. We took a survey area of $20 \mathrm{deg}^{2}$ and a total observation time of $2900 \mathrm{hr}$. This example shows that Ly $\alpha$ EoR science is well within the reach of our modest template instrument. The calculated sensitivities achieved on the deep fields are sufficient to detect Ly $\alpha$ in broad $\Delta k / k$ bins ranging from $k=0.01$ to $10 h \mathrm{Mpc}^{-1}$ in both clustering and Poisson fluctuations.

Ideally the spectral resolution would match the maximum $k$ available in the angular direction; however, higher spectral resolution requires longer integration times needed to realize photon noise limited sensitivity, which tends to degrade the instrument sensitivity. The angular resolution does not affect surface brightness sensitivity directly, but it does determine the depth to which lower-redshift galaxies may be masked using a deep ancillary continuum galaxy survey. Although the continuum emission from galaxies can in principle be removed by looking at the signal across the frequency direction, as explained before, contamination from other lines at lower redshifts does pose a problem to the detection of the Ly $\alpha$ signal, in particular from the $\mathrm{H} \alpha$ line. The most straightforward way to remove this contamination would be by masking the pixels where these low- $z$ galaxies are found, either from the observation itself or using another, high-sensitivity, continuum observation. For this approach, the angular resolution of the Ly $\alpha$ experiment has to be good enough in order to have enough pixels left after the masking. Therefore, this instrument is required to have higher angular resolution than spectral resolution. Figure 15 also shows the expected contamination from the $\mathrm{H} \alpha$ line from galaxies at $z=0.5$ (black dots). This was calculated following the same approach as for the Ly $\alpha$ line and using the H $\alpha$-to-SFR relation taken from Kennicutt et al. (1994) and Kennicutt (1998b). Removing low- $z$ galaxies down to a mass of $\sim 6.6 \times 10^{10} M_{\odot}$, corresponding to a cut in luminosity $L>10^{42} \mathrm{erg} \mathrm{s}^{-1}$, would bring this contamination below the Ly $\alpha$ signal (black crosses). Using the $\mathrm{H} \alpha \mathrm{LF}$ from Geach et al. (2010) normalized to the SFRD at $z=0.5$, we get an expected angular density of about $25 \mathrm{H} \alpha$ emitters per square degree per band, which would mean that only $\sim 0.98 \%$ of the pixels would be masked.

Note that the rejection of interloping low-redshift galaxies requires a full treatment that is beyond the scope of this paper. Foreground rejection may also be significantly enhanced by simultaneously detecting additional EoR spectral features beyond Ly $\alpha$, which are produced by interlopers with very low probability. Combining these Ly $\alpha$ measurements with other EoR observations $\left(\mathrm{CO}, \mathrm{C}+\right.$, and particularly $\left.\mathrm{H}_{\mathrm{I}} 21 \mathrm{~cm}\right)$ offers additional information on EoR star formation, metallicity, and ionization history. The possibility of constructing an experiment in a near-IR band to measure the Ly $\alpha$ flux in order to correlate it with the $21 \mathrm{~cm}$ signal was also explored by Wyithe et al. (2007). Although they used simple models to estimate the fluctuations in each of these two lines, they also considered several foregrounds that will contaminate the observations and concluded that it is possible to remove enough foregrounds that the intensity of radiation emitted from galaxies can be constrained from the cross-correlation.

\section{SUMMARY}

In this paper, we took into account the main contributions to Ly $\alpha$ emission from recombinations, collisions, continuum emission in galaxies, and scattering of Lyn photons to calculate the intensity of Ly $\alpha$ emission from galaxies and from the IGM during the EoR.

We started by theoretically calculating the intensities using astrophysical data from several observational results and then implemented the calculation in a simulation using a modified version of the code SimFast 21 to obtain the spatial fluctuations of Ly $\alpha$ emission. The simulation allowed us to calculate the Ly $\alpha$ emission taking into account the spatial fluctuations of the different astrophysical parameters, which represents an improvement over theoretical calculations that only use the average values.

Our simulations showed that to achieve optical depths compatible with the WMAP constraints, the high SFRD required implies that for reasonable values of UV and Ly $\alpha$ escape fraction the intensity of $\operatorname{Ly} \alpha$ emission from galaxies is dominant over the emission from the IGM.

By testing different SFR halo mass parameterizations, we constrained the intensity of $\mathrm{Ly} \alpha$ emission from galaxies to be about $(1.43-3.57) \times 10^{-8}$ and (4.55-9.73) $\times$ $10^{-11} \mathrm{erg}^{-1} \mathrm{~cm}^{-2} \mathrm{sr}^{-1}$ at redshifts 7 and 10 , respectively, which is dominant over the intensity of $\mathrm{Ly} \alpha$ emission from the IGM at $z=7$ (about $1.6 \times 10^{-5}$ ) but less at $z=10$ $\left(1.1 \times 10^{-10} \mathrm{erg} \mathrm{s}^{-1} \mathrm{~cm}^{-2} \mathrm{sr}^{-1}\right)$. Since the intensity levels we found are lower than the extragalactic background intensity from galaxies and so are too low to be detected with an experiment aiming the absolute background intensity, we propose an intensity mapping experiment that will allow us to measure the Ly $\alpha$ power spectrum.

For reasonable astrophysical conditions the process of hydrogen reionization was done by UV radiation originated in galaxies with luminosities below the high-redshift observational threshold. In this work, we showed the different ways by which UV emission is connected to $\operatorname{Ly} \alpha$ emission, and so we stress how 
it would be useful to use intensity mapping of Ly $\alpha$ emission to probe the overall intensity of UV radiation.

Ly $\alpha$ emission can also be connected to the $21 \mathrm{~cm}$ signal from the EoR, since the continuum photons above the Ly $\alpha$ line that redshift to this line in the IGM contribute to the radiative coupling of the $21 \mathrm{~cm}$ signal to the gas temperature. The cross-correlation of the $\mathrm{Ly} \alpha$ and the $21 \mathrm{~cm}$ lines can be used to reduce systematics and foregrounds encountered with $21 \mathrm{~cm}$ observations. In particular, the discontinuity of the cross-correlation power spectra will provide constraints in the evolution of the IGM ionized fraction.

In previous studies, we have discussed the use of $\mathrm{CO}$ molecular and $\mathrm{C}_{\mathrm{II}}$ fine-structure atomic lines to complement $21 \mathrm{~cm}$ data in the attempt to probe the IGM during reionization. Our study shows that Ly $\alpha$ intensity mapping is also a viable approach to probe reionization and is within experimental reach over the coming decade.

This work was supported by FCT-Portugal with the grant SFRH/BD/51373/2011 for M.B.S. and under grant PTDC/ FIS/100170/2008 for M.B.S. and M.G.S.

A.C. and Y.G. acknowledge support from NSF CAREER AST-0645427 and NASA NNX10AD42G at UCI.

M.B.S. was a long-term Visiting Student at UCI, supported by NSF CAREER AST-0645427, when this work was initiated, and she thanks the Department of Physics and Astronomy at UCI for hospitality during her stay.

\section{REFERENCES}

Barkana, R., \& Loeb, A. 2001, PhR, 349, 125

Barkana, R., \& Loeb, A. 2005, ApJ, 626, 1

Bouwens, R. J., Illingworth, G. D., Labbe, I., et al. 2011, Natur, 469, 504 Bouwens, R. J., Illingworth, G. D., Oesch, P. A., et al. 2012a, ApJL, 752, 5 Bouwens, R. J., Illingworth, G. D., Oesch, P. A., et al. 2012b, ApJ, 754, 83 Boylan-Kolchin, M., Springel, V., White, S. D. M., Jenkins, A., \& Lemson, G. 2009, MNRAS, 398, 1150

Brown, R. L., \& Mathews, W. G. 1970, ApJ, 160, 939

Cantalupo, S., Porciani, C., \& Lilly, S. J. 2008, ApJ, 672, 48

Chen, X., \& Miralda-Escudé, J. 2008, ApJ, 684, 18

Chuzhoy, L., \& Zheng, Z. 2007, ApJ, 670, 912

Conroy, C., \& Wechsler, R. H. 2009, ApJ, 696, 620

Cooray, A., Gong, Y., Smidt, J., \& Santos, M. G. 2012, ApJ, 756, 92

Davé, R., Cen, R., Ostriker, J. P., et al. 2001, ApJ, 552, 473

Dayal, P., Ferrara, A., \& Saro, A. 2010, MNRAS, 402, 1449

De Lucia, G., \& Blaizot, J. 2007, MNRAS, 375, 2

Dijkstra, M., Haiman, Z., \& Spaans, M. 2006a, ApJ, 649, 14

Dijkstra, M., Haiman, Z., \& Spaans, M. 2006b, ApJ, 649, 37

Dopita, M. A., \& Sutherland, R. S. 2003, Astrophysics of the Diffuse Universe (Berlin: Springer)

Fan, X., Strauss, M. A., Becker, R. H., et al. 2006, AJ, 132, 117

Fardal, M. A., Katz, N., Gardner, J. P., et al. 2001, ApJ, 562, 605

Fernandez, E. R., \& Komatsu, E. 2006, ApJ, 646, 703

Fernández-Soto, A., Lanzetta, K. M., \& Chen, H.-W. 2003, MNRAS, 342, 1215

Forero-Romero, J. E., Yepes, G., Gottlöber, S., et al. 2011, MNRAS, 415, 3666 Furlanetto, S. R., Oh, S. P., \& Briggs, F. H. 2006, PhR, 433, 181 Geach, J. E., Cimatti, A., Percival, W., et al. 2010, MNRAS, 402, 1330

Gnedin, N. Y., Kravtsov, A. V., \& Chen, H.-W. 2008, ApJ, 672, 765
Gong, Y., Cooray, A., Silva, M., et al. 2012, ApJ, 745, 49

Gong, Y., Cooray, A., Silva, M. B., Santos, M. G., \& Lubin, P. 2011, ApJL, 728,46

Gould, A., \& Weinberg, D. H. 1996, ApJ, 468, 462

Guo, Q., White, S., Boylan-Kolchin, M., et al. 2011, MNRAS, 413, 101

Haardt, F., \& Madau, P. 2012, ApJ, 746, 125

Haiman, Z., Spaans, M., \& Quataert, E. 2000, ApJL, 537, 5

Hayes, M., Schaerer, D., Östlin, G., et al. 2011, ApJ, 730, 8

Iye, M., Ota, K., Kashikawa, N., et al. 2006, Natur, 443, 186

Jensen, H., Laursen, P., Mellema, G., et al. 2013, MNRAS, 428, 1366

Jiang, L., Egami, E., Kashikawa, N., et al. 2011, ApJ, 743, 65

Karzas, W. J., \& Latter, R. 1961, ApJS, 6, 167

Kashikawa, N., Shimasaku, K., Malkan, M. A., et al. 2006, ApJ, 648, 7

Kennicutt, R. C., Jr. 1998a, ARA\&A, 36, 189

Kennicutt, R. C., Jr. 1998b, ApJ, 498, 541

Kennicutt, R. C., Jr., Tamblyn, P., \& Congdon, C. E. 1994, ApJ, 435, 22

Komatsu, E., Smith, K. M., Dunkley, J., et al. 2011, ApJS, 192, 18

Larson, D., Dunkley, J., Hinshaw, G., et al. 2011, ApJS, 192, 16

Latif, M. A., Schleicher, D. R. G., Spaans, M., \& Zaroubi, S. 2011, MNRAS, 413, L33

Lehnert, M. D., Nesvadba, N. P. H., Cuby, J.-G., et al. 2010, Natur, 467, 940

Lidz, A., Furlanetto, S. R., Oh, S. P., et al. 2011, ApJ, 741, 70

Madau, P., \& Pozzetti, L. 2000, MNRAS, 312, L9

Maraston, C. 2005, MNRAS, 362, 799

Martin, P. G. 1988, ApJS, 66, 125

Mesinger, A., \& Furlanetto, S. 2007, ApJ, 669, 663

Mesinger, A., \& Haiman, Z. 2007, ApJ, 660, 923

Mitra, S., Choudhury, T. R., \& Ferrara, A. 2012, MNRAS, 419, 1480

Oesch, P. A., Bouwens, R. J., Illingworth, G. D., et al. 2012, ApJ, 745, 110

Ota, K., Iye, M., Kashikawa, N., et al. 2008, ApJ, 677, 12

Ota, K., Iye, M., Kashikawa, N., et al. 2010, ApJ, 722, 803

Ouchi, M., Shimasaku, K., Akiyama, M., et al. 2008, ApJS, 176, 301

Ouchi, M., Shimasaku, K., Furusawa, H., et al. 2010, ApJ, 723, 869

Pawlik, A. H., Schaye, J., \& van Scherpenzeel, E. 2010, in ASP Conf. Ser. 432, New Horizons in Astronomy: Frank N. Bash Symposium 2009, ed. L. M.

Stanford, J. D. Green, L. Hao, \& Y. Mao (San Francisco, CA: ASP), 230

Pengelly, R. M. 1964, MNRAS, 127, 145

Popesso, P., Biviano, A., Rodighiero, G., et al. 2012, A\&A, 537, A58

Razoumov, A. O., \& Sommer-Larsen, J. 2010, ApJ, 710, 1239

Salvaterra, R., Della Valle, M., Campana, S., et al. 2009, Natur, 461, 1258

Santos, M. G., Ferramacho, L., Silva, M. B., Amblard, A., \& Cooray, A. 2010, MNRAS, 406, 2421

Santos, M. G., Silva, M. B., Pritchard, J. R., Cen, R., \& Cooray, A. 2011, A\&A, 527, A93

Santos, M. R. 2004, MNRAS, 349, 1137

Schaerer, D. 2002, A\&A, 382, 28

Sheth, R. K., \& Tormen, G. 1999, MNRAS, 308, 119

Shibuya, T., Kashikawa, N., Ota, K., et al. 2012, ApJ, 752, 114

Shimasaku, K., Kashikawa, N., Doi, M., et al. 2006, PASJ, 58, 313

Shull, J. M., \& van Steenberg, M. E. 1985, ApJ, 298, 268

Shull, M., Harness, A., Trenti, M., \& Smith, B. 2012, ApJ, 747, 100

Siana, B., Teplitz, H. I., Colbert, J., et al. 2007, ApJ, 668, 62

Smith, B. D., Hallman, E. J., Shull, J. M., \& O'Shea, B. W. 2011, ApJ, 731, 6

Springel, V., White, S. D. M., Jenkins, A., et al. 2005, Natur, 435, 629

Steidel, C. C., Bogosavljević, M., Shapley, A. E., et al. 2011, ApJ, 736, 160

Taniguchi, Y., Ajiki, M., Nagao, T., et al. 2005, PASJ, 57, 165

Visbal, E., \& Loeb, A. 2010, JCAP, 11, 16

Wang, X., Tegmark, M., Santos, M. G., \& Knox, L. 2006, ApJ, 650, 529

Wise, J. H., \& Cen, R. 2009, ApJ, 693, 984

Wyithe, S., Loeb, A., \& Schmidt, B. 2007, arXiv:0705.1825

Yajima, H., Choi, J.-H., \& Nagamine, K. 2011, MNRAS, 412, 411

Zahn, O., Reichardt, C. L., Shaw, L., et al. 2012, ApJ, 756, 65

Zaldarriaga, M., Colombo, L., Komatsu, E., et al. 2008, arXiv:0811.3918

Zheng, Z., Cen, R., Trac, H., \& Miralda-Escudé, J. 2010, ApJ, 716, 574

Zheng, Z., Cen, R., Weinberg, D., Trac, H., \& Miralda-Escudé, J. 2011, ApJ, 739,62 